\title{
SNLS spectroscopy: testing for evolution in type la supernovae ${ }^{\star}$
}

T. J. Bronder ${ }^{1, \star \star}$, I. M. Hook ${ }^{1}$, P. Astier ${ }^{2}$, D. Balam ${ }^{3}$, C. Balland ${ }^{2}$, S. Basa ${ }^{4}$, R. G. Carlberg ${ }^{5}$, A. Conley ${ }^{5}$, D. Fouchez ${ }^{6}$, J. Guy ${ }^{2}$, D. A. Howell ${ }^{5}$, J. D. Neill ${ }^{7}$, R. Pain $^{2}$, K. Perrett ${ }^{5}$, C. J. Pritchet ${ }^{3}$, N. Regnault ${ }^{2}$, M. Sullivan ${ }^{5}$, S. Baumont ${ }^{2}$, S. Fabbro ${ }^{8}$, M. Filliol ${ }^{4}$, S. Perlmutter ${ }^{9}$, and P. Ripoche ${ }^{6}$

1 University of Oxford Astrophysics, Denys Wilkinson Building, Keble Road, Oxford OX1 3RH, UK e-mail: timothy.bronder@kirtland.af.mil

2 LPNHE, CNRS-IN2P3 and University of Paris VI \& VII, 75005 Paris, France

3 Department of Physics and Astronomy, University of Victoria, PO Box 3055, Victoria, BC V8W 3P6, Canada

${ }^{4}$ LAM, BP 8, Traverse du Siphon, 13376 Marseille Cedex 12, France

Department of Astronomy and Astrophysics, University of Toronto, 50 St. George Street, Toronto, ON M5S 3H4, Canada

${ }^{6}$ CPPM, CNRS-Luminy, Case 907, 13288 Marseille Cedex 9, France

SRL California Institute of Technology, 1200 East California Blvd., Pasadena CA 91125, USA

8 CENTRA - Centro Multidisciplinar de Astrofísica, IST, Avenida Rovisco Pais, 1049 Lisbon, Portugal

9 University of California, Berkeley and Lawrence Berkeley National Laboratory, Mail Stop 50-232, Lawrence Berkeley National Laboratory, 1 Cyclotron Road, Berkeley CA 94720, USA

Received 16 April 2007 / Accepted 31 July 2007

\section{ABSTRACT}

\begin{abstract}
Aims. We present a quantitative study of a new data set of high redshift Type Ia supernovae spectra, observed at the Gemini telescopes during the first 34 months of the Supernova Legacy Survey. During this time 123 supernovae candidates were observed, of which 87 have been identified as SNe Ia at a median redshift of $z=0.720$. Spectra from the entire second year of the survey and part of the third year (59 total SNe candidates with 46 confirmed SNe Ia) are published here for the first time. The spectroscopic measurements made on this data set are used determine if these distant SNe comprise a population similar to those observed locally.

Methods. Rest-frame equivalent width and ejection velocity measurements are made on four spectroscopic features. Corresponding measurements are presented for a set of 167 spectra from 24 low- $z$ SNe Ia from the literature.

Results. We show that there exists a sample at high redshift with properties similar to nearby SNe. The high- $z$ measurements are consistent with the range of measurements at low- $z$ and no significant difference was found between the distributions of measurements at low and high redsift for three of the features. The fourth feature displays a possible difference that should be investigated further. Correlations between Type Ia SNe properties and host galaxy morphology were also found to be similar at low and high $z$, and within each host galaxy class we see no evidence for redshift-evolution in SN properties. A new correlation between SNe Ia peak magnitude and the equivalent width of SiII absorption is presented. Tests on a sub-set of the SNLS SNe demonstrates that this correlation reduces the scatter in SNe Ia luminosity distances in a manner consistent with the lightcurve shape-luminosity corrections that are used for Type Ia SNe cosmology.

Conclusions. We show that this new sample of SNLS SNe Ia has spectroscopic properties similar to nearby objects.
\end{abstract}

Key words. supernovae: general - cosmology: observations - surveys

\section{Introduction}

Hubble diagrams making use of the "standardizeable" nature of Type Ia SNe (Riess et al. 1998; Perlmutter et al. 1999; Knop et al. 2003; Astier et al. 2006) indicate the significant presence of a "dark energy" that is driving the accelerating expansion of the universe. This result is additionally constrained by observations of the power spectrum of the cosmic microwave backround (Spergel et al. 2003, 2006), properties of massive clusters (Allen et al. 2002) and other large scale structure observations (Seljak et al. 2005). The equation of state parameter of this dark energy (defined as $w=p / \rho$, the ratio of dark energy pressure $p$ to density $\rho$ ) has been investigated by recent surveys (Knop et al. 2003; Barris et al. 2004; Riess et al. 2004), but the results are still

\footnotetext{
* Tables 6, Appendices A and B are are only available in electronic form at http://www . aanda.org

$\star \star$ Current address: AFRL Directed Energy 3550 Aberdeen Ave SE, Kirtland AFB, Albuquerque, NM 87117.
}

consistent with a wide range of dark energy models. The CFHT Supernova Legacy Survey (SNLS, Astier et al. 2006) aims to increase the measured precision of this parameter by finding and following about 500 distant Type Ia SNe over a five-year survey lifetime.

The fundamental assumptions of the methods used to estimate the cosmological parameters with $\mathrm{SNe}$ are that the selected low $z$ and high $z$ objects have similar peak magnitudes (i.e. within the locally observed dispersion) and that the established lightcurve shape-luminosity relations are applicable to these $\mathrm{SNe}$ at all redshifts. However, the current understanding of SNe Ia explosions (see Hillebrandt \& Niemeyer 2000, for a review) and their progenitors (PGs) illustrates that there may be some differences, or evolution, in their inherent properties at high redshifts (Hoflich et al. 1998; Hatano et al. 2000; Lentz et al. 2000). Such effects could distort the cosmological results of SNe Ia surveys. The differences in properties of SNe in hosts of different morphology (Wang et al. 1997; Umeda et al. 1999; 
Hamuy et al. 2000; Howell 2001; Sullivan et al. 2003; Gallagher et al. 2005; Sullivan et al. 2006a) provides additional evidence of SNe Ia heterogeneity. The luminosity-lightcurve shape calibrations used for SNe Ia cosmology (Riess et al. 1996; Phillips et al. 1999; Goldhaber et al. 2001; Guy et al. 2005) make an empirical accomodation for the dispersion of SNe properties observed locally, but these methods may be vulnerable to systematic changes in the SNe Ia population at high redshifts (Leibundgut 2001). Thus, it is imperative to undertake a quantitative investigation to determine whether there are any changes in these objects that may affect their reliability as distance indicators for cosmology.

The spectra of SNe Ia reveal more subtle characteristics than photometric observations and are well suited for this investigation. For example, measurements of photospheric ejection velocities $\left(v_{\mathrm{ej}}\right)$ explore the kinetic energy and distribution of elements in the expanding SN ejecta. These measurements provided some of the first evidence of intrinsic differences between Type Ia SNe (Branch et al. 1988) and have also been shown to correlate with luminosity (Wells et al. 1994; Fisher et al. 1995). More recently, Benetti et al. (2005) used cluster analysis of measurements of the decline rate of SNe Ia $v_{\mathrm{ej}}$ to yield a new way of grouping low $z$ SNe that perhaps illuminates the role of different explosion mechanisms in SNe Ia diversity. Comparisons of SNe Ia ejection velocities from objects at different redshifts have also been made (e.g. Hook et al. 2005; Blondin et al. 2006; Balland et al. 2006) demonstrating that the range of velocities measured in SNe Ia ejecta are comparable at all redshifts. Folatelli (2004, F04 hereafter) illustrated the utility of equivalent width $(E W)$ measurements on low- $z$ SNe Ia spectra and Garavini et al. (2007) extended this work to compare 12 high- $z$ objects to the low $z$ population. Hachinger et al. (2006) also illustrated correlations between these $E W$ measurements and the velocitygradient classification scheme from Benetti et al. (2005). Branch et al. (2006) presents additional evidence for a temperature or explosion mechanism sequence in $\mathrm{SNe}$ Ia with comparisons of different low- $z$ objects in $E W$-space.

These studies all illustrate how quantitative spectroscopic measurements can be used to explore the factors that affect the heterogeneity and homogeneity of SNe Ia. The utility of these parameters for exploring differences in high- $z$ SNe Ia is limited, however, as the majority of these measurements cannot be reproduced in the high- $z$ data. These limitations are due to the reduced signal-to-noise $(\mathrm{S} / \mathrm{N})$, redshift effects (which shift the features to redder regions where sky noise is dominant or moves features out of optical detection limits entirely), host galaxy contamination and the smaller number of observations which all characterise high- $z$ data.

The analysis presented here includes rest frame equivalent width and ejection velocity measurements on a large set of $\mathrm{SNe}$ Ia spectra observed for the SNLS with methods that are specifically tailored for high- $z$ data. These measurements are similar to the recently published work of Garavini et al. (2007) but are utilized on a much larger high- $z$ set and include a different treatment of host galaxy contamination and estimates of the low $-z$ trends. The studied set of SNe were selected from the objects considered "confirmed" SNe Ia by the SNLS (see Howell et al. 2005, H05 hereafter) and used in the survey's cosmological analysis. These high- $z$ results are compared to corresponding measurements on a set of low- $z$ supernovae from the literature. The results of this investigation address the cosmological implications from high- $z$ SNe surveys by determining whether this SNLS-set of distant objects comprises a population that is spectroscopically similar to their low- $z$ counterparts. An overview of the data sets used in this analysis is presented in Sect. 2. The methods for measureing $E W$ and $v_{\mathrm{ej}}$ are presented in Sect. 3. The results and a comparison of the two $\mathrm{SNe}$ sets are presented in Sect. 4 , in Sect. 5 these results are discussed in more detail and the conclusions are presented in Sect. 6 .

\section{Spectroscopic data sets - overview}

A large number of the $\mathrm{SNe}$ Ia that were used to provide the latest constraints on $w$ and other cosmological parameters (Astier et al. 2006; Spergel et al. 2006) are studied in this paper. Specifically, this includes the SNLS objects observed at the Gemini telescopes during the first year of the survey. This set is augmented by Gemini spectra from the entire 2nd year and part of the 3rd year of SNLS observations, which are published here for the first time. This section briefly describes the observation, data reduction, and identification of these SNLS SNe spectra in Sect. 2.1. In Sect. 2.2 a summary of the set of low- $z$ SNe that were quantitatively compared to the distant $\mathrm{SNe}$ Ia is provided.

\subsection{High redshift SNLS supernovae}

\subsubsection{SNLS spectra - observation, data reduction and classification}

Full details of the methods used for SNLS SN candidate selection and observation can be found in Sullivan et al. (2006a) and $\mathrm{H} 05$, respectively. In summary, the Canada-France-Hawaii Telescope Legacy Survey (CFHTLS), using the Megacam wide field imager, acquires real time lightcurves of possible $\mathrm{SNe}$ by repeatedly imaging four, one square-degree fields approximately every four days (observers' frame) in several filters (a combination of $g^{\prime}, r^{\prime}, i^{\prime}$, and $z^{\prime}$ ) as part of the Deep component of the CFHTLS. Spectroscopic observations are then made at $8 \mathrm{~m}$-class telescopes in order to identify the SN-type and redshift of these candidates.

The objects studied here were observed at the Gemini North and South telescopes with the Gemini Multi Object Spectrograph (GMOS; Hook et al. 2004) in longslit mode. Typically, three $20 \mathrm{~min}$ to $30 \mathrm{~min}$ exposures are taken of each candidate. The GMOS R400 grating (400 lines per mm) and $0.75^{\prime \prime}$ slit are used, which gives a $6.9 \AA$ spectral resolution. A central wavelength of $680 \mathrm{~nm}$ was chosen for objects at an estimated $z \lesssim 0.4$ and objects at higher estimated $z$ are observed with a central wavelength setting of $720 \mathrm{~nm}$. This central wavelength selection is important in order to ensure that the defining SiII features (located at rest wavelengths of $\sim 4100 \AA$ and $\sim 6150 \AA$ ) of SNe Ia spectra are observed. The $680 \mathrm{~nm}$ setting was paired with the GG455_G0305 order-sorting filter to provide wavelength coverage from $4650 \AA$ to $8900 \AA$. The $720 \mathrm{~nm}$ setting was used along with the OG515_G0306 order-sorting filter to cover wavelengths from $5100 \AA$ to $9300 \AA$. Queue observing was employed to observe the SNLS candidates, which require better than $0.75^{\prime \prime}$ image quality (corrected to zenith), in either classical or nod-and-shuffle $(\mathrm{N} \& \mathrm{~S})$ observing modes (see H05).

The reduction of the spectra was completed using IRAF $^{1}$, independently from that of $\mathrm{H} 05$, but with very similar methods.

1 IRAF is written and supported by the IRAF programming group at the National Optical Astronomy Observatories (NOAO). NOAO is operated by the Association of Universities for Research in Astronomy (AURA), Inc. under cooperative agreement with the National Science Foundation. 

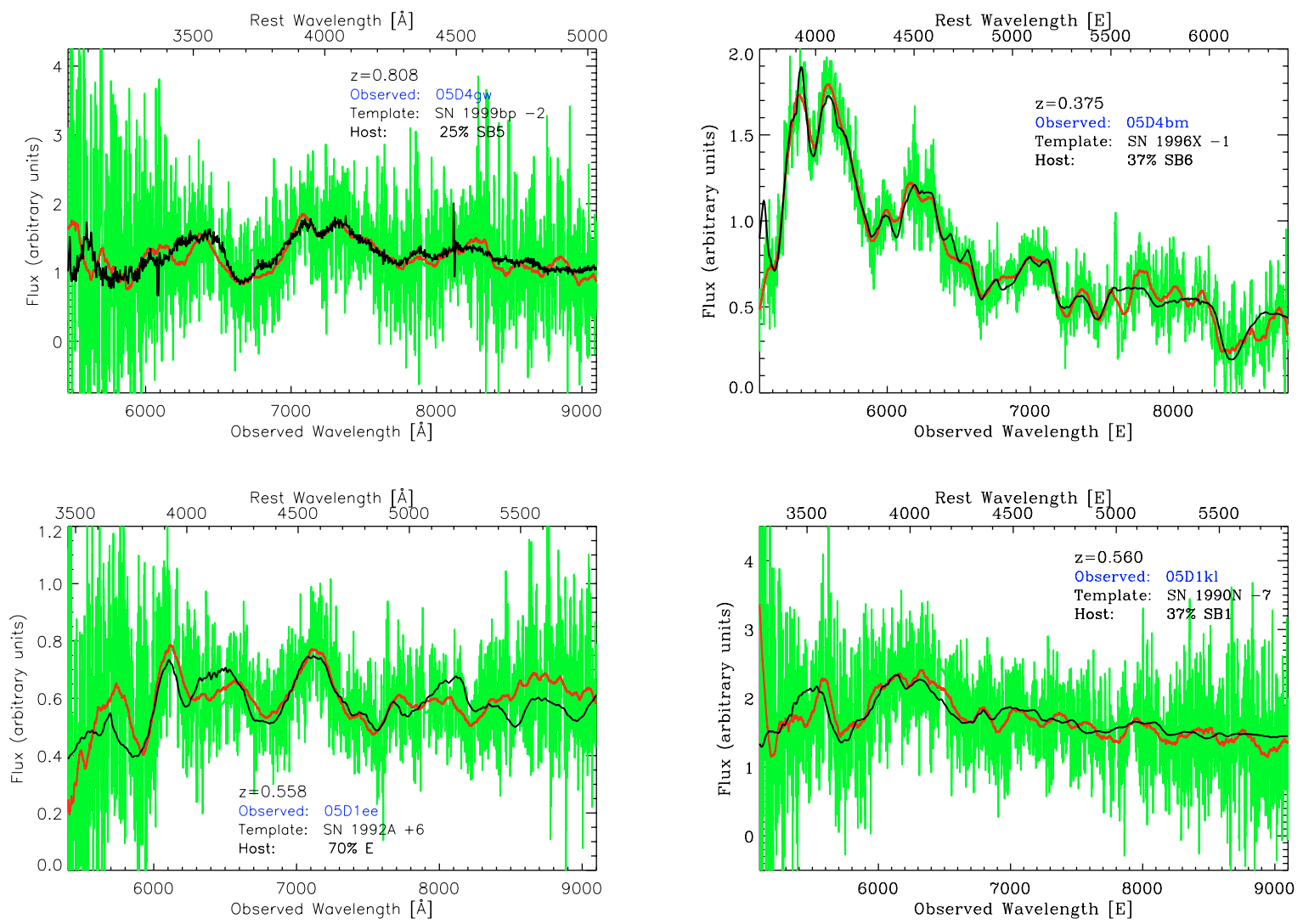

Fig. 1. Example spectra of SNLS candidates observed by Gemini. The observed spectra after subtraction of the best-fitting host galaxy template are displayed in green. The smoothed spectra are shown in red and the best fitting template SNe are overplot in black. The top two objects are "certain type Ia" SNe $(\mathrm{CI}=5)$ as they have clear signs of SiII. The bottom objects are SNLS candidates with CI $=4($ left $)$ and $\mathrm{CI}=3($ right $)$. These spectra match SNe Ia templates but have ambiguous SiII absorption. All of the other spectra are available online.

The independent reductions were made to check for any systematic differences in the pipelines and the results from all reductions were in very close agreement.

To classify the SNLS objects, the final spectra are quantitatively compared to template supernovae from large library of low- $z$ objects with a $\chi^{2}$ matching program (see Howell \& Wang 2002, and H05 for details). The results from this matching program and the opinions of SNLS members are used to rank the identifications according to one "confidence index" (CI) from the six categories defined in H05, with a 5 being a "Certain Ia" and a 0 as "Not a Ia". Objects that are ranked at a CI of 3 or greater are considered "confirmed" Type Ia SNe. These are the objects that were used in the cosmology fits from Astier et al. (2006) and also make up the 80 high- $z$ SNe Ia studied here.

The selection criteria of the SNLS sample means that Type Ia $\mathrm{SNe}$ at high- $z$ with properties that are very different from those seen nearby are likely to be rejected. Thus in the comparisons below the existence of a new population of high- $z \mathrm{SNe}$ with unusual properties cannot be ruled out. However as will be seen later, the selection process does allow a range of SNIa properties through, and thus tests for evolution within this range are possible.

\subsubsection{Summary of the SNLS dataset}

The SNe candidates observed during the first year of the survey at the Gemini telescopes were published in H05. Table 2 of H05 lists the derived properties of these spectra. This set includes 64 candidates observed from August 2003 through October 2004. Within this set, 41 objects were classified with as confirmed $\mathrm{SNe}$ with a $\mathrm{CI} \geq 3$ and are studied here.

Spectra from the entire second year and part of the third year of SNLS observations at Gemini are presented here for the first time. These spectra were observed between November 2004 and May 2006. During this period 59 SNe candidates were observed and 46 of these objects were confirmed as SNe Ia. The setup and conditions of the observations of these objects are listed online in Table 6. The derived properties and final classifications of these spectra are summarized in Table 7. Some example spectra are presented in Fig. 1 and all of the spectra are available in the online version of this paper.

For the analysis in this paper we make use of not only spectral properties but also photometric properties. The latter are derived from lightcurve fits to the SNLS CFHT photometry, following the methods described in Astier et al. (2006). The main lightcurve parameters used are the date of $B$-band maximum light, and percentage increase relative to the host galaxy (interpolated to the date of spectroscopy).

\subsection{Low redshift type la supernovae}

\subsubsection{Low-z spectra}

The spectra that make up the low redshift sample in this study were selected from the literature. A majority of these spectra can be found on the online Supernova Spectrum Archive 
Table 1. The low redshift spectra analyzed in this study. The dates correspond to the epoch relative to maximum luminosity in the $B$-band. Many of these spectra are available in the SUSPECT archive as well as their listed publications.

\begin{tabular}{cll}
\hline \hline Name & Date(s) & Reference \\
\hline 1981B & 0,20 & Branch et al. (1983) \\
1986G & $-5,-4,-3,0$ & Phillips et al. (1987) \\
1989B & $-7,-5,-3,-2,-1,3,5,8,9,11,12,13,16,17,18,19$ & Barbon et al. (1990), Wells et al. (1994) \\
1990N & $-14,-7,2,3,17$ & Mazzali et al. (1993) \\
1991M & 3 & Gomez \& Lopez (1998) \\
1991T & $-12,-11,-10,-8,-7,-6,0,10,15$ & Mazzali et al. (1995) \\
1991bg & $0,1,2$ & Turatto et al. (1996) \\
1992A & $-5,-1,3,5,6,7,9,11,16,17$ & Kirshner et al. (1993) \\
1994D & $-11,-10,-9,-8,-7,-5,-4,-2,2,4,10,11,12,13,20$ & Patat et al. (1996) \\
1994Q & 10 & Gomez \& Lopez (1998) \\
1996X & $-2,-1,0,1,2,7$ & Salvo et al. (2001) \\
1997br & $-9,-8,-7,-6,-4,8$ & Li et al. (1999) \\
1997cn & 3 & Turatto et al. (1998) \\
1998aq & $-8,0,1,2,3,4,5,6,7$ & Branch et al. (2003) \\
1998bu & $-4,-3,-2,8,9,10,11,12,13$ & Capellaro et al. (2001) \\
1999aa & $-11,-3,-1,5,6$ & Garavini et al. (2004) \\
1999ac & $-15,-9,0,2,8,11$ & Garavini et al. (2004) \\
1999aw & $3,5,5,12$ & Strolger et al. (2002) \\
1999by & $-5,-4,-3,-2,3,4,5,6,7,8,11$ & Garnavich et al. (2004) \\
1999ee & $-11,-9,-8,-6,-4,-2,-1,0,3,5,7,8,9,12,14$ & Hamuy et al. (2002) \\
2000E & $-2,3,5,14$ & Valentini et al. (2003) \\
2000cx & $-4,-3,-2,-1,0,1,5,6,7,9,11$ & Li et al. (2001) \\
2002bo & $-14,-13,-11,-7,-6,-5,-4,-3,-2,-1,4$ & Benetti et al. (2004) \\
2003du & $-11,-7,13$ & Anupama et al. (2005) \\
\hline
\end{tabular}

Table 2. The photometric properties of the low- $z$ spectra analyzed in this study. The published photometry from these objects was re-fit to determine the magnitudes and lightcurve shape parameters shown here. SN 1991M did not have published photometry in $B$-band, so the parameters for this object were not determined. The SNLS models did not fit the sub-luminous SN 1991bg with any accuracy; the values given here for this unusual object are from Altavilla et al. (2004). The precision of the derived values for the unusual SN 2000cx are also in doubt and were not used in any of the analysis in this paper. Uncertainties in each quantity are given in brackets.

\begin{tabular}{|c|c|c|c|c|c|c|}
\hline Name & $z$ & $\mathrm{MJD}_{\max }^{a}$ & $m_{B}^{b}$ & $(B-V)_{\max }^{c}$ & $s^{d}$ & $M_{B}^{e}$ \\
\hline 1981B & 0.0060 & $44671.84(0.09)$ & $11.83(0.01)$ & $-0.06(0.01)$ & $0.95(0.01)$ & $-19.58(0.22)^{1}$ \\
\hline $1986 \mathrm{G}$ & 0.0027 & $46560.53(0.05)$ & $11.91(0.01)$ & $0.83(0.01)$ & $0.73(0.01)$ & \\
\hline 1989B & 0.0024 & $47564.39(0.27)$ & $12.12(0.02)$ & $0.30(0.02)$ & $0.94(0.01)$ & $-19.03(0.31)^{1}$ \\
\hline $1990 \mathrm{~N}$ & 0.0034 & $48081.92(0.03)$ & $12.53(0.01)$ & $-0.09(0.01)$ & $1.07(0.01)$ & $-19.59(0.23)^{1}$ \\
\hline $1991 \mathrm{M}$ & 0.0072 & & & & $\ldots$ & \\
\hline $1991 \mathrm{~T}$ & 0.0060 & $48374.25(0.04)$ & $11.30(0.01)$ & $-0.04(0.01)$ & $1.05(0.01)$ & $-20.07(0.27)^{2}$ \\
\hline $1991 \mathrm{bg}$ & 0.0030 & $48604.50(1.0)$ & $14.75(0.04)$ & $0.00(0.08)$ & $.68(0.10)$ & \\
\hline $1992 \mathrm{~A}$ & 0.0063 & $48639.71(0.02)$ & $12.41(0.01)$ & $-0.09(0.01)$ & $0.85(0.01)$ & $-18.73(0.22)^{7}$ \\
\hline 1994D & 0.0015 & $49431.41(0.01)$ & $11.64(0.01)$ & $-0.19(0.01)$ & $0.84(0.01)$ & $-19.44(0.22)^{7}$ \\
\hline 1994Q & 0.0294 & $49494.35(0.90)$ & $16.16(0.09)$ & $-0.14(0.04$ & $1.11(0.04)$ & $\ldots$ \\
\hline 1996X & 0.0070 & $50190.65(0.10)$ & $12.86(0.01)$ & $-0.12(0.01)$ & $0.90(0.01)$ & $-19.38(0.22)^{7}$ \\
\hline $1997 \mathrm{br}$ & 0.0053 & $50558.57(0.03)$ & $13.23(0.02)$ & $0.12(0.01)$ & $0.89(0.01)$ & $\ldots$ \\
\hline $1997 \mathrm{cn}$ & 0.0162 & $50593.36(0.32)$ & $18.12(0.02)$ & $0.92(0.01)$ & $0.89(0.02)$ & $\ldots$ \\
\hline 1998aq & 0.0037 & $50930.16(0.02)$ & $12.18(0.01)$ & $-0.25(0.01)$ & $0.98(0.01)$ & $-19.87(0.23)^{6}$ \\
\hline 1998bu & 0.0030 & $50951.89(0.04)$ & $11.97(0.01)$ & $0.15(0.01)$ & $0.99(0.01)$ & $-19.18(0.26)^{1}$ \\
\hline 1999aа & 0.0144 & $51231.82(0.03)$ & $14.59(0.01)$ & $-0.18(0.01)$ & $1.12(0.01)$ & $\ldots$ \\
\hline $1999 \mathrm{ac}$ & 0.0095 & $51250.07(0.08)$ & $13.99(0.01)$ & $-0.08(0.01)$ & $1.02(0.01)$ & $\ldots$ \\
\hline 1999aw & 0.0380 & $51253.34(0.08)$ & $16.59(0.01)$ & $-0.17(0.01)$ & $1.22(0.01)$ & $\ldots$ \\
\hline 1999ee & 0.0114 & $51468.89(0.01)$ & $14.69(0.01)$ & $0.11(0.01)$ & $1.05(0.01)$ & $-19.70(0.30)^{3}$ \\
\hline $2000 \mathrm{e}$ & 0.0047 & $51576.44(0.03)$ & $12.68(0.01)$ & $0.01(0.01)$ & $1.03(0.01)$ & $-20.08(0.25)^{7}$ \\
\hline $2000 \mathrm{cx}$ & 0.0079 & $51752.03(0.03)$ & $12.92(0.01)$ & $-0.09(0.01)$ & $0.91(0.01)$ & \\
\hline 2002bo & 0.0042 & $52356.43(0.04)$ & $13.82(0.01)$ & $0.29(0.01)$ & $0.95(0.01)$ & $-19.04(0.46)^{7}$ \\
\hline $2003 \mathrm{du}$ & 0.0064 & $52766.22(0.19)$ & $13.36(0.01)$ & $-0.25(0.01)$ & $1.02(0.01)$ & $-19.53(0.45)^{5}$ \\
\hline
\end{tabular}

${ }^{a}$ Date of maximum luminosity (rest-frame $B^{\prime}$ );

${ }^{b}$ Maximum apparent magnitude in rest-frame $B^{\prime}$ magnitudes, corrected for Milky Way extinction with $E(B-V)$ values from Schlegel et al. (1998);

${ }^{c} B-V$ color at ( $B$-band) maximum luminosity;

${ }^{d}$ Stretch values, based on fitting a fiducial $s=1.0$ Type Ia template;

${ }^{e}$ Absolute peak luminosities $\left(B^{\prime}\right)$, not adjusted for lightcurve shape-luminosity corrections. These made use of the following distance modulus references - 1: Gibson et al. (2000); 2: Saha et al. (2001); 3: Stritzinger et al. (2002); 4: Candia et al. (2003); 5: Anupama et al. (2005); 6: Riess et al. (2005); 7: Tully (1988). 
$\left(\right.$ SUSPECT $\left.^{2}\right)$. The main goal of this study is to quantify the similarities and differences between high- $z$ SNe Ia and those discovered locally, so objects with epoch and wavelength coverage that approximated that of the SNLS sample were selected. These spectra were corrected for peculiar velocities and redshift where appropriate. All of the low $z$ objects that met this criteria are listed in Table 1.

The low- $z$ sample includes 167 spectra from 24 different objects. The set includes the so-called "core normal" (Branch et al. 2006) objects (i.e. SNe similar to SN 1981B, Branch et al. 1983; SN 1989B, Barbon et al. 1990; Wells et al. 1994; SN 1992A, Kirshner et al. 1993; or SN 1994D, Patat et al. 1996) as well as spectroscopically peculiar $\mathrm{SNe}$ Ia such as the overluminous 1991T-like objects and underluminous 1991bg-like SNe.

\subsubsection{Low- $z$ photometry and distance estimation}

The photometric properties of these nearby objects are listed in Table 2. These properties have been presented in the literature (Perlmutter et al. 1999; Phillips et al. 1999; Altavilla et al. 2004; Reindl et al. 2005) with slightly different results depending on the color corrections used or the lightcurve fits to the published photometry. To remove systematic differences when comparing results, the photometry from these SNe was re-fit by SNLS members to derive the magnitudes and lightcurve shape parameters used later in this analysis. This lightcurve fitting used a new template spectrum for K-corrections (see Hsiao et al. (in press) and Conley et al. (in prep.) for full details). The parameters derived from these fits are summarized in Table 2.

These re-derived photometric properties were also used to estimate the absolute peak $\left(B^{\prime}\right)$ magnitudes, $M_{\mathrm{B}_{\text {peak }}}$, of the low$z$ SNe Ia. A new estimate of these magnitudes is of interest as the published values have (in general, see Phillips et al. 1999; and Altavilla et al. 2004; for exceptions) already been corrected for a derived lightcurve shape-luminosity correction. In order to fully explore the homogeneity of $\mathrm{SNe}$ at different redshifts and to investige any possible spectroscopic sequences in $\mathrm{SNe} \mathrm{Ia}$, an uncorrected, or intrinsic, $M_{\mathrm{B}_{\text {peak }}}$ is desired.

To make this estimation, the observed magnitudes from Table 2 were first corrected for host galaxy reddening using the relation between $\Delta m_{15}(B)$ (estimated from the re-fit lightcurves) and $B-V$ colour described by Eq. (7) in Phillips et al. (1999). Combined with the relations in Eqs. (9) and (11) from the same publications, the observed and estimated "true" $B-V$ colours were approximated to constrain the host galaxy reddening for each SN. This is the only step of the estimate that made use of a SNe Ia lightcurve shape-luminosity correction and is unavoidable as some assumption of the intrinsic colour of these objects is necessary to infer the host galaxy reddening. This reddening was then converted to a $B^{\prime}$-extinction with $R_{B}=3.0$. This is not the standard value used in this exercise and does not take into account extremely red objects (Elias-Rosa et al. 2006), but it is approximately the median value of the different $R_{B}$ estimates in the literature. The selection of this $R_{B}$ value does not have a large effect on the results in this paper. A different $R_{B}$ would systematically shift all the values in Table 2 a slight amount, but leave the relative relationship between the magnitude of the different $\mathrm{SNe}$ the same, which is the true parameter of interest in this study as illustrated in Fig. 10.

Next, the distance modulus, $\mu$, to the host galaxy was used to convert to an absolute magnitude via $\mu=m-M$ (where $m$ and $M$ are the observed and absolute magnitudes, respectively).

${ }^{2}$ http://bruford.nhn.ou.edu/suspect/index 1.html

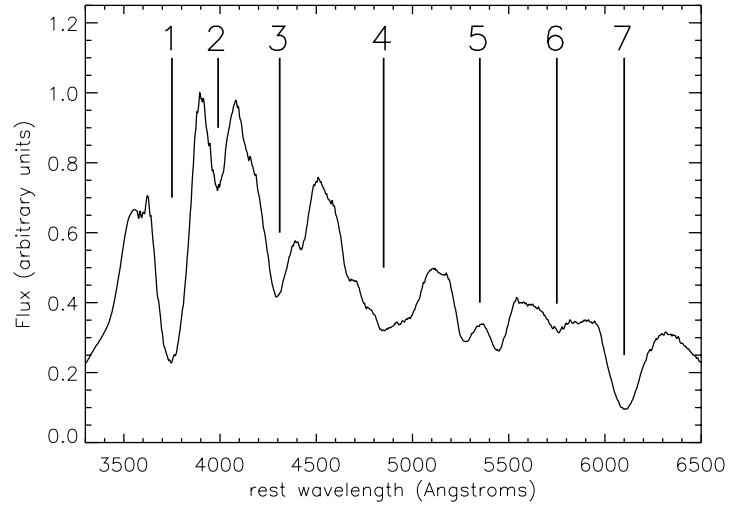

Fig. 2. The spectroscopic regions of specific interest to SNe Ia $E W$ measurements, as defined by F04, are shown here on a core normal $\mathrm{SN}$ Ia near maximum light. The wavelength ranges and nomenclature for these features is summarized in Table 3.

Table 3. Wavelength regions for the SNeIa features defined by F04.

\begin{tabular}{clcc}
\hline \hline $\begin{array}{c}\text { Feature } \\
\text { Number }\end{array}$ & Label & $\begin{array}{c}\text { Blue-ward } \\
\text { Limit }(\AA)\end{array}$ & $\begin{array}{c}\text { Red-ward } \\
\text { Limit }(\AA)\end{array}$ \\
\hline 1 & "CaII H\&K" & $3500-3800$ & $3900-4100$ \\
2 & "SiII 4000" & $3900-4000$ & $4000-4150$ \\
3 & "MgII" & $3900-4150$ & $4450-4700$ \\
4 & "FeII" & $4500-4700$ & $5050-5550$ \\
5 & "SII W" & $5150-5300$ & $5500-5700$ \\
6 & "SiII 5800" & $5550-5700$ & $5800-6000$ \\
7 & "SiII 6150" & $5800-6000$ & $6200-6600$ \\
\hline
\end{tabular}

To yield the most correct distance modulus, the authors used the most reliable Cepheid variable based distances to the $\mathrm{SNe}$ host galaxies that were available in the literature. These distances were available for 9 of the SNe in Table 1 and another 5 low- $z$ SNe had reliable host distances from the Nearby Galaxies Catalogue (Tully 1988). The remaining objects did not have any independent distance constraints and were not considered for these $M_{B}$ estimates. The errors in each of these steps were propagated through this estimate to provide a final error on $M_{B}$. The final column in Table 2 lists these intrinsic peak magnitude values for the 14 low- $z$ SNe that had reliable, independent distance estimates and secure observed photometric properties.

\section{Spectroscopic analysis techniques}

\subsection{Equivalent widths}

Rest frame equivalent width measurements are a shapeindependent method of quantifying the strength of spectroscopic features. The method used in this $E W$ analysis is similar to F04 and Garavini et al. (2007), which defined eight regions that contain the strongest absorption features observed in core normal $\mathrm{SNe}$ Ia spectra near maximum luminosity. The first seven regions are illustrated in Fig. 2, and the nomenclature and wavelength ranges used to define them are given in Table 3. For supernovae, the continuum needed to make the $E W$ measurement is not well defined, so it is estimated by making a straight line fit between the local maxima that bound each feature. The following rules govern how the continuum is set:

- the pseudo-continuum is always set within the feature regions listed in Table 3; 
- the feature boundaries are selected to maximise the wavelength span of the measurement without intersecting the boundaries of any neighboring features;

- the $E W$ is always calculated in the rest frame.

The continuum is set automatically but a human judgement is made on its placement to ensure the guidelines above are followed. The $E W$ is then calculated by numerically integrating underneath this "pseudo-continuum" at every wavelength pixel $\lambda_{i}$ (for all $N$ points within the set continuum bounds):

$E W=\Sigma_{i=1}^{N}\left(1-\frac{f_{\lambda}\left(\lambda_{i}\right)}{f_{c}\left(\lambda_{i}\right)}\right) \Delta \lambda_{i}$

where $f_{\lambda}$ is the flux level of the spectrum, and $f_{c}$ is the flux of the pseudo-continuum. The statistical uncertainty in the $E W$ measurement is calculated by propagating the estimated uncertainties in the flux and pseudo-continuum:

$\sigma_{E W}^{2}=\Sigma_{i=1}^{N}\left(\left\{\frac{\sigma_{f}\left(\lambda_{i}\right)}{f_{c}\left(\lambda_{i}\right)}\right\}^{2}+\left\{\frac{f_{\lambda}\left(\lambda_{i}\right)}{f_{c}\left(\lambda_{i}\right)^{2}}\right\}^{2} \cdot \sigma_{c_{i}}^{2}\right) \Delta \lambda_{i}^{2}$

here $\sigma_{f}$ is the measurement uncertainty in the flux and $\sigma_{c_{i}}$ is the uncertainty in the pseudo-continuum flux. The $\sigma_{c_{i}}$ is calculated via the propagation of the correlated errors in the selection and calculation of this continuum.

In addition to the error calculated in Eq. (2), uncertainties can be caused by variations in the extent or the slope of the pseudo-continuum. The range and effects of these possible pseudo-continuum variations were estimated by randomly shifting the continuum boundaries within $\frac{R}{4} \AA$ of the selected maxima (where $R$ is the range of the feature boundaries, see Fig. 2) for the feature and spectrum in question. The $E W$ was then re-measured, and the standard deviation of these measurements (which were repeated 50 times) was accepted as an approximation of this error in each $E W$ measurement. For the high $\mathrm{S} / \mathrm{N}$ spectra, the possible variations in the continuum were found to be the dominant source of error. The high- $z$ data was subject to some additional systematic errors, so this general procedure had to be amended for these objects; this is discussed in the following section.

In calculating the low- $z E W$, any possible effects from noise fluctuations were mitigated by setting the continuum end-points to an average flux value that was computed over a $25 \AA$ window around the selected maxima. Observed error spectra were not available for most of the objects in this nearby sample, so the statistical error could not be calculated. However, the dominant error from the possible pseudo-continuum variations was estimated as described above. In total, these uncertainties were on the order of $5-10 \%$ of the measured low- $z E W$. This measurement techniqe differs slightly from previously published $E W$ studies (F04; Hachinger et al. 2006; Branch et al. 2006), but the results here are in very good agreement with these other references. Based on the wavelength coverage available in the high- $z$ spectra, this $E W$ analysis is focused on $E W$ features 1,2 , and 3 - CaII H\&K, SiII 4000, and MgII in the F04 nomenclature ( $E W\{\mathrm{CaII}\}, E W\{\mathrm{SiII}\}$, and $E W\{\mathrm{MgII}\}$ hereafter).

\subsection{High-z EW measurements}

As explained in Sect. 3.2.1, the high- $z$ spectra were median filtered in order to set the feature bounds. The $E W$ measurement was then made with the initial, un-filtered points as Fig. 3 shows. For the SNLS data set, the available statistical uncertainty was

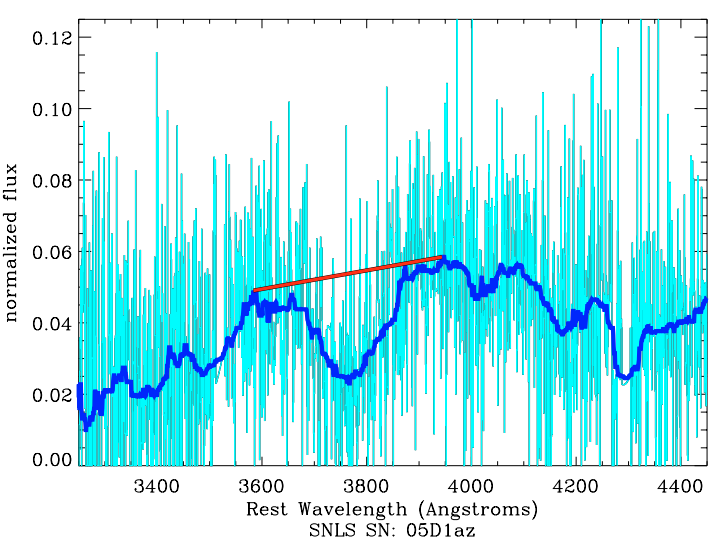

Fig. 3. An example of an $E W$ measurement (for $E W\{\mathrm{CaII}\}$ in this case) on a high- $z$ SNLS object. The median filtered spectrum is plotted on top of the observed spectrum. The local maxima of the feature have been found within this median filter. The $E W$ is then computed by numerically integrating the weighted, un-filtered flux underneath the pseudocontinuum defined by the two maxima.

used to weight each point so that the equivalent width was calculated as

$E W_{\mathrm{SNLS}}=\left(\Sigma_{i=1}^{N}\left(1-\frac{f_{\lambda}\left(\lambda_{i}\right)}{f_{c}\left(\lambda_{i}\right)}\right) \Delta \lambda_{i} w_{i}\right) \frac{N}{\Sigma\left(w_{i}\right)}$

where $w_{i}$ is the weighting function at each point:

$w_{i}=1 / \sigma_{f}\left(\lambda_{i}\right)^{2}$.

The statistical errors were calculated as defined in Eq. (2). The error induced by any variance in the pseudo-continuum was computed with the method described in Sect. 3.1. A description of the errors caused by sky noise and host galaxy contamination is presented in the next sections.

Note that if either the lower or upper range of an $E W$ measurement (defined in Table 3) was outside of the observed wavelength range then the measurement had to be removed from further analysis. This constraint affected results on a measurement-by-measurement basis and caused the removal of $61 \mathrm{EW}$ measurements in the SNLS data set. This left a total of 217 measurements from 73 different high- $z$ SNe in the final set of $E W$ results. A number of these measurements were later removed for host contamination considerations, however (see Sect. 3.2.2).

\subsubsection{High-z EW systematic errors I - signal to noise}

The sky noise affects the high- $z$ measurements by augmenting the local maxima selected to set the pseudo-continuum. This can result in an overestimate of the equivalent width. A median filter was used to correct this systematic effect. The size of the filter window was selected via simulations in which spectra from the low- $z$ sample were degraded with noise to match that in the high$z$ SNe. The $E W$ was then re-calculated on each of the artificial spectra, using a median filter set to various window sizes. Using a median filter over a $60 \AA$ window proved to be the most robust for all measurements at the wide range of $\mathrm{S} / \mathrm{N}$ levels covered by the high- $z$ spectra.

These simulations were also used to check that the uncertainties in $E W$ measurements behaved as expected for a given signal-to-noise input spectrum. Both the simulations and the final measurement results demonstrated that the statistical errors 
in the high- $z$ sample were approximately $7 \%-20 \%$ of the measured $E W$ value. This is nearly twice the uncertainty of the low- $z$ objects due to their reduced $\mathrm{S} / \mathrm{N}$.

\subsubsection{High-z EW systematic errors II - host galaxy contamination}

In nearby objects, the angular separation between the host and $\mathrm{SN}$ candidate is enough that most observations are not affected by host galaxy light. This is not the case with distant $\mathrm{SNe}$. In previous high redshift spectroscopic studies, this error has been addressed by subtracting host galaxy templates during the $\chi^{2}$-fitting process (Howell \& Wang 2002; Hook et al. 2005; Garavini et al. 2007), during the extraction of the SN spectrum (Blondin et al. 2006), or not at all. In reality, the host contamination tends to "wash out" the absorption troughs in $\mathrm{SNe}$ spectra, which causes the measured strength of these features to be lower than what would be seen in a spectrum free of host galaxy flux. The actual effects of the contamination are dependent on the redshift and host galaxy type of each object in addition to the amount of contamination. The rolling search method of the SNLS provides extensive photometric data that was used to estimate the amount of host galaxy contamination at the epoch of spectroscopy for each SN.

Each object in the SNLS sample has epochs of non-detection before and after the explosion. These are used as a baseline from which to calculate the precentage increase of the SN light over that of the host galaxy on each date when photometry was taken. A $1.10^{\prime \prime}$ (diameter) circular aperture centred on the $\mathrm{SN}$ is used as it is the basis for the percent contamination estimates that the SNLS photometric pipeline provides for each SN candidate. By interpolating these measurements to the date of spectroscopy, the amount of host contamination in the supernova spectrum can be estimated.

In order to do this, the fact that the spectroscopic aperture is not the same size and shape as the photometric aperture must be taken into account. The spectroscopic aperture is rectangular, with a width of $0.75^{\prime \prime}$ and a length set by the extraction window used during the spectroscopic data reduction. The amount of host light entering the slit was simply scaled from that in the $1.10^{\prime \prime}$ photometric aperture by assuming a spatially flat profile. The amount of SN light entering the slit was estimated by modelling the SN signal as a two-dimensional PSF with a FWHM equal to the seeing in the appropriate GMOS acquisition image. The photometrically determined percent increase can thus be converted to a "percent contamination" of host flux in the observed spectrum.

The effects of host contamination on the $E W$ results was determined by artificially contaminating low $z$ spectra from several different SNe Ia with template galaxy spectra from E, S0, Sa, Sc, and starburst galaxies. In each run of the simulation, the range of the observed CFHT $i^{\prime}$-band was shifted, based the redshift of each SNLS object, to where it would fall in the rest frame. The $i^{\prime}$-band was chosen because this is the wavelength region where SNe Ia are brightest for the redshift range of this survey; most of the SNLS observations are thus made in this band. The flux of a chosen host galaxy template was then scaled to match the level host contamination (estimated as described above) and added to the low- $z$ supernova spectrum. The $E W$ was re-measured to give the percent error caused by the specific host type and contamination amount for every $E W$ measurement on each SNLS object.

The results of these simulations were then used to correct for this systematic error in the high- $z$ spectra. The median value of the percent error estimates among the different low $-z$ spectra was used as the best estimate for the contamination effects on each SNLS supernova, $E W$ measurement, and galaxy type in question. The uncertainty of this percent error estimate was quantified with the standard deviation of the contamination effects from different host galaxy and SNe templates. The effects within each general host galaxy type were similar, so this uncertainty was only $5 \%-7 \%$ of the measured $E W$. Including the excess statistical uncertainty from reduced $\mathrm{S} / \mathrm{N}$, this error means that the uncertainties in the high- $z E W$ results are roughly 2-3 times those in the corresponding low $z$ set. The galaxy type for the $\mathrm{SNe}$ was selected based on the observed host features in each affected SNLS spectrum. The results of these corrections on the measured $E W$ values are displayed in Fig. 5.

The results of these simulations helped determine that objects with greater than $65 \%$ host contamination were too biased to be included in the final analysis of this data set. This is because at this contamination level, a majority of the high- $z E W$ results require adjustments greater than $100 \%$ of the measured $E W$ and this amount of host flux severely alters the shape of the $\mathrm{SNe}$ spectra so that the local maxima for each feature cannot be identified in a manner consistent with the rest of the sample. This removed measurements from 19 objects (in addition to the measurements removed for observed-wavelength restrictions). The final set of high- $z$ results thus includes a total $162 \mathrm{EW}$ measurements from 54 SNLS SNe Ia.

\subsection{Ejection velocities}

The distribution of the ions in the homologously expanding SN photosphere and the kinetic energy of the thermonuclear explosion are expressed in the ejection velocities measured in the SN spectrum. Measurements of the ejection velocities of the features attributed to CaII H\&K, SII (near 25640) and SiII (near $\lambda 6150$ ) - our $E W$ features 1, 5, and 7, respectively - have previously been studied in both nearby (Branch et al. 1988; Wells et al. 1994; Fisher et al. 1995; Mazzali et al. 1998; Benetti et al. 2005; Mazzali et al. 2005) and high redshift (Hook et al. 2005; Blondin et al. 2006) SNe Ia populations. The redshifts of the distant objects that make up the bulk of the SNLS sample are high enough that the SII "W" ( 25640$)$ and SiII $\lambda 6150$ features are generally not observed, so only the CaII H\& K feature was studied.

The ejection velocity, $v_{\mathrm{ej}}$, was calculated as the blueshift of the minimum of the feature away from an estimated rest wavelength of $3945.0 \AA$ (a weighted average of the two components of the CaII H\&K feature). The contribution of other ions to the absorption in the wavelength range of CaII H\&K (most notably $\mathrm{SiII}$ ), as well the intrinsic distribution of CaII across velocity space, can give this feature a "kinked" shape with two possible minima. This shape makes it difficult to determine where the mininum of the feature is located. This difficulty is compounded by the low $\mathrm{S} / \mathrm{N}$ of the high- $z$ objects. To make a consistent measurement between the two sets, the entire CaII H\&K feature was fit with a Gaussian function. The best fit was estimated with robust, non-linear least-squares MPFIT $^{3}$ routine in IDL. The fit was weighted to the most dominant minima of the absorption region by making small, initial Gaussian fits to the deepest, bluest, part of the feature. The wavelength range from this initial fit was then expanded iteratively to span the entire absorption

\footnotetext{
${ }^{3}$ see http://cow.physics.wisc. edu/ craigm/idl/
} 

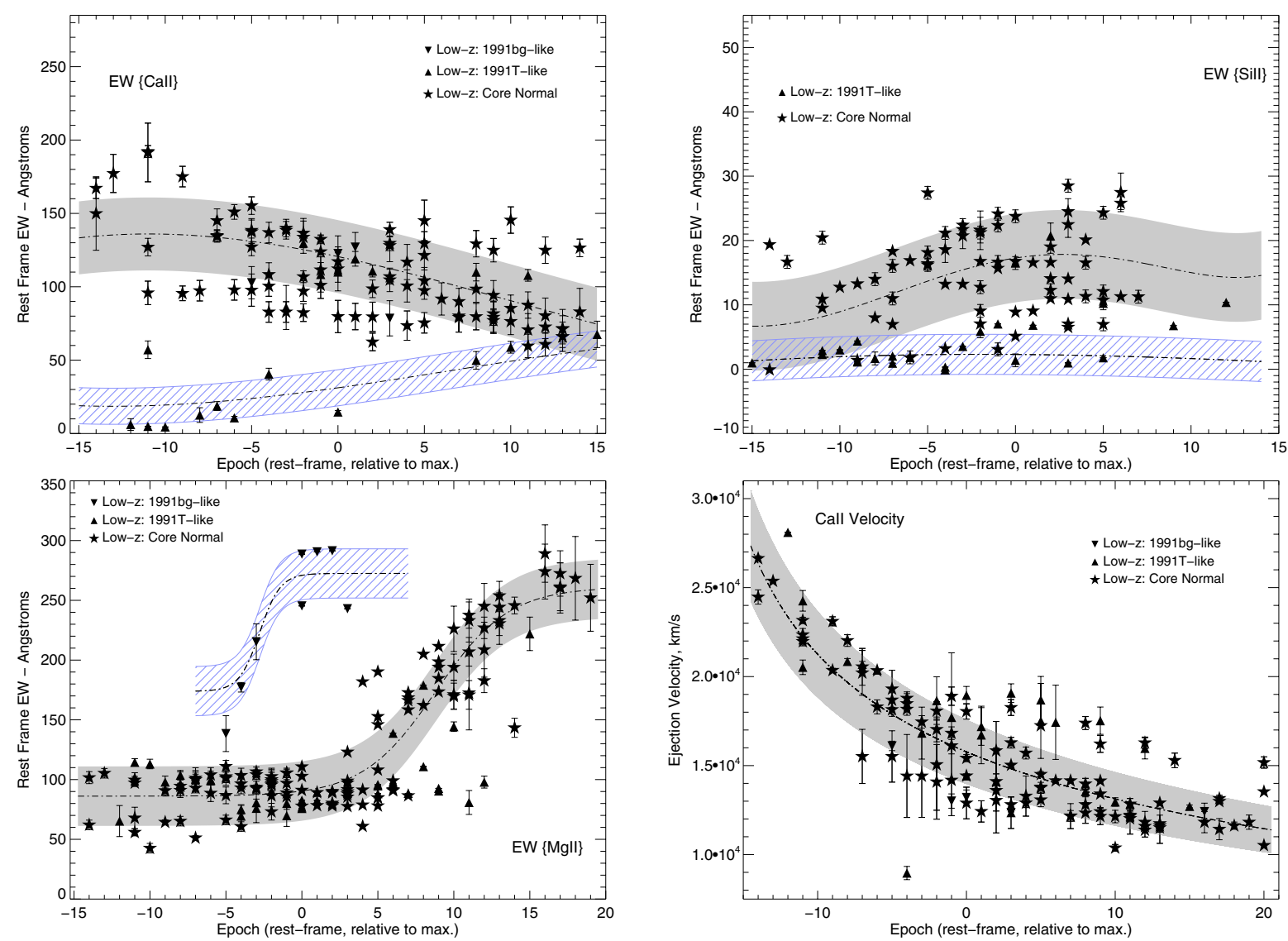

Fig. 4. EW measurements for the "CaII H\&K" (top, left), "SiII" (top, right), and "MgII" (bottom, left) features and CaII H\&K vej (bottom, right) plotted versus rest-frame epoch, for the low- $z$ sample. The shaded, grey region marks the mean trend and $1 \sigma$ distribution of the epoch evolution of these measurements in core normal objects. The striped region marks the same trend for 1991T-like objects in the $E W\{\mathrm{CaII}\}$ and $E W\{\mathrm{SiII}\}$ plots and the trend for $1991 \mathrm{bg}$-like objects in the $E W\{\mathrm{MgII}\}$ plot. These trends were estimated with $E W$ measurements on template SNe Ia spectra from the literature.

trough, and the minimum of the final fit was used to calculate $v_{\text {ej }}$ as

$v_{\mathrm{ej}}=-c \cdot\left(\frac{\left(\frac{\lambda_{\mathrm{m}}-\lambda_{0}}{\lambda_{0}}+1\right)^{2}-1}{\left(\frac{\lambda_{\mathrm{m}}-\lambda_{0}}{\lambda_{0}}+1\right)^{2}+1}\right)$

where $\lambda_{\mathrm{m}}$ is the measured featured minimum, $\lambda_{0}$ is $3945.0 \AA$ and $c$ is the speed of light. The statistical error for this fit was computed by propagating the uncertainties in the final Gaussian parameters into Eq. (5). An additional source of error, caused by uncertainty in the selection of points used to fit the Gaussian curves, was estimated by taking the difference between the calculated velocities from the initial and final fits.

Without having measurement uncertainties available for the low $-z$ spectra, the statistical errors were not calculated for these objects. For the high- $z$ data, the lower $\mathrm{S} / \mathrm{N}$ meant these spectra had to be smoothed before the Gaussian fits to the CaII H\&K feature could be made. Again a $60 \AA$ median filter was used. The filtered flux was fit with a Gaussian model that was weighted by the error spectra. The statistical error in the final $v_{\mathrm{ej}}$ values was estimated by propagating the estimated redshift uncertainties through the velocity calculation.

No corrections were necessary to account for the systematic error from host galaxy contamination as this error was minimal for $v_{\mathrm{ej}}$ measurements made on the $\mathrm{SNe}$ within the $65 \%$ contamination limit discussed in Sect. 3.2.2.

\section{Spectroscopic analysis results}

\subsection{Low-z results}

The results of the low- $z E W$ measurements, plotted against restframe epoch (relative to $B^{\prime}$ maximum light) are shown in the first 3 panels in Fig. 4 . The mean trend (and $1 \sigma$ deviation) exhibited by normal SNe Ia for each feature is shown in the grey contour in each panel. This trend was derived by making $E W$ measurements on a template core normal Type Ia SN spectrum made from a combination of core normal spectra by Nugent et al. (2002). The mean trends for the 1991T-like and 1991bg-like objects were derived from similarly constructed template Type Ia spectra (Nugent et al. 2002; Stern et al. 2004; Levan et al. 2005, Nugent private communication) and are shown where appropriate. The $1 \sigma$ deviation shown on these trends was estimated by expanding the error bars on each trend until a fit to the appropriate low $-z$ SNe measurements produced a reduced $\chi^{2}$ of 1.0 . This was done as other methods of estimating the $1 \sigma$ range would be more dependent on the varying epoch and object sampling of the low- $z$ data set. A constant scatter as a function of epoch was thus assumed, hence the lack of variance over epoch in Figs. 4-6.

The core normal objects exhibit fairly homogenous behavior in these $E W$ plots. Not surprisingly, this parameter space also clearly differentiates between the SNe Ia sub-types. The lack of absorption from IME (intermediate mass elements) in 1991Tlike objects (which is due to inferred higher temperatures during thermonuclear runaway in these objects) is clearly seen in 

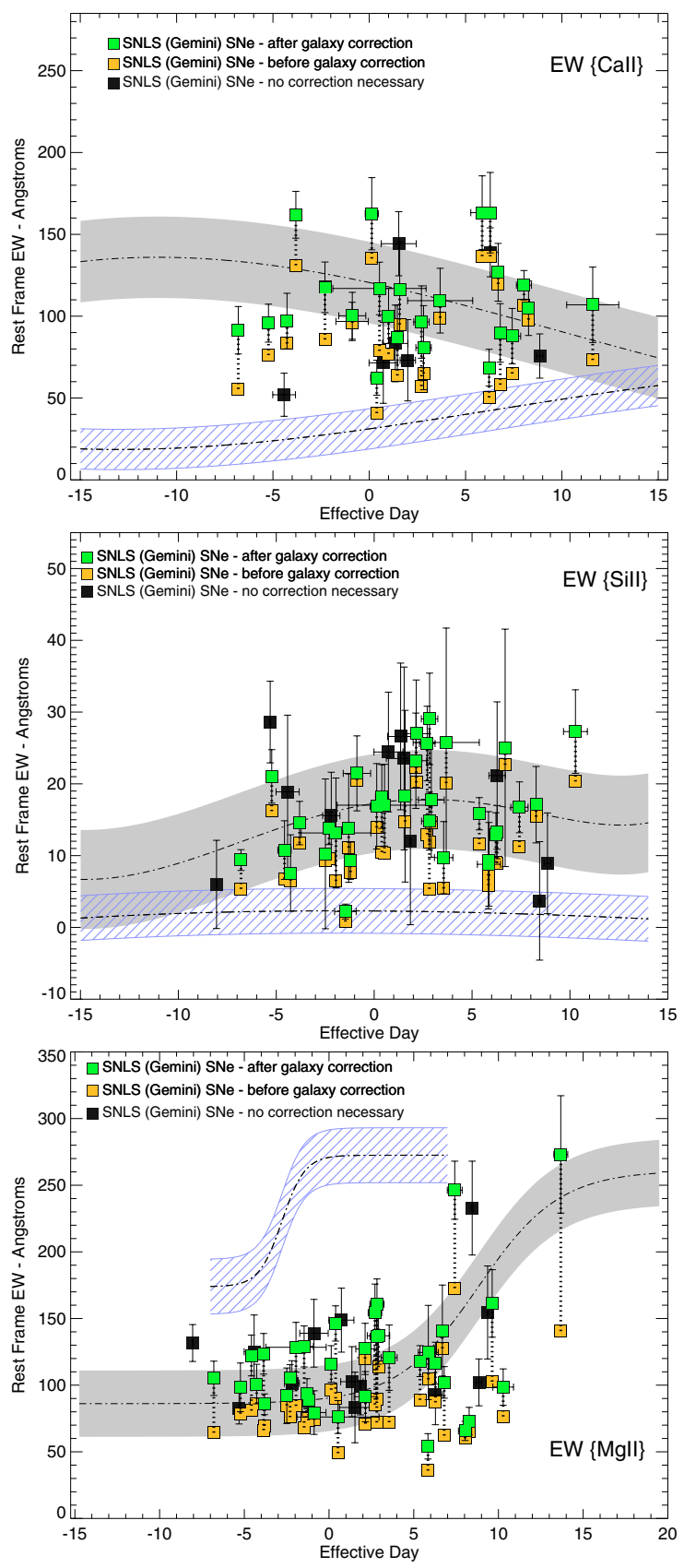

Fig. 5. Measurements of the $E W$ of the "CaII H\&K", "SiII", and "MgII" features (from top to bottom), versus rest-frame epoch, in the SNLS high- $z$ sample. The shaded and striped regions are the same as in Fig. 4. The $E W$ measurements that did not need any correction for the systematic effects of host galaxy contamination are shown with the dark, filled squares. All of the other points illustrate the measurements that were corrected for host contamination. The adjusted $E W$ values are connected to the corresponding initial measurements with dotted lines.

the $E W\{\mathrm{CaII} H \& \mathrm{~K}\}$ and $E W\{\mathrm{SiII}\}$ results. Similarly, the additional absorption from (primarily) TiII in cooler, 1991bg-like objects results in a significant difference with regards to $E W\{\mathrm{MgII}\}$ measurements. These results are in agreement with the qualitative and quantitative differences that have already been established for these different $\mathrm{SNe}$ sub-types (Filippenko et al. 1992a,b; Benetti et al. 2005; Branch et al. 2006).

The changes of CaII $\mathrm{H} \& \mathrm{~K} v_{\mathrm{ej}}$ with rest-frame epoch is shown in the fourth panel of Fig. 4. The mean trend was estimated by fitting a power law to all of the data points from core normal objects. The core normal objects display a broad trend about this best fit and the SN sub-types are generally interspersed around it. Previous estimations of SN Ia ejection velocities implied that over-luminous objects have significantly higher velocities (Jeffrey et al. 1992), but the statistical analysis of SiII velocities by Benetti et al. (2005) suggests that these objects perhaps differ in their velocity gradients, which were not studied here as corresponding measurements are not available on the high- $z$ set.

\subsection{High-z results and comparison to low-z SNe}

The $E W$ measurements on the high- $z$ data before and after correction for host galaxy contamination are shown in Fig. 5. The corrected results are compared to the corresponding low- $z$ results in Fig. 6 and all of these high- $z$ results are listed in Table A. 1 in the Appendix.

Figure 6 shows that the general trends exhibited by the low $-z$ sample are indeed followed by the high redshift objects and all of the SNLS results are within the range of the low- $z$ SNe measurements. A few spectra (marked with open circles in Fig. 6) had $E W$ results and other characteristics that are more similar to the SN 1991T-like outliers; these objects are discussed in more detail in Sect. 4.3.

The fraternity between the high redshift SNe measurements and the trends exhibited by the local, core normal objects was further investigated with $\chi^{2}$ tests. This test compared the high- $z$ measurements to the core normal low redshift "model" illustrated for each feature in Figs. 4-6. When making these $\chi^{2}$ calculations, the full uncertainties for each measurement were used. The $1 \sigma$ dispersion in the low- $z$ trends was also considered by adding this error (in quadrature) to the uncertainty of each high$z$ measurement. The uncertainty in the estimated epoch for every high- $z$ result was also added (again, in quadrature) according to the low $-z$ trend for each measurement. The results, shown in Table 4, indicate that there are no statistically significant differences between the spectroscopic features of these SNe Ia for three of the parameters studied here.

The exception to this result is the comparison of $E W\{\mathrm{MgII}\}$ measurements; however, the reality of this difference is questionable. It is possible that this difference is a statistical artifact caused by the differences in the epoch sampling and total number of objects between the high- $z$ and low $-z$ data sets. Figure 4 shows that there are an appreciable number of low- $z$ core normal points between 0 and +10 days past maximum (where the bulk of the outliers in the SNLS set are located) which define the $1 \sigma$ range on the empirically derived mean trend. Yet these points are from 15 different SNe only; half of the number of SNLS objects in the same range. This sampling difference, combined with the fact that the low- $z$ sample is not well defined (i.e. it is not a complete sample) could mean that the true amount of variance in this feature is underestimated in the low $z$ mean trends, which would artificially increase the significance of the measured difference in the high- $z$ results. If this epoch selection effect is not responsible for the difference then it is interesting to consider possible physical interpretations, which we discuss in Sect. 5.

\subsection{High-z results: identification of SNe la sub-types}

The comparisons in Fig. 6 show that a handful of the high$z$ SNLS SNe Ia exhibit spectroscopic features that are more consistent with the $1991 \mathrm{~T}$ or $1991 \mathrm{bg}$-like SNe from the low $-z$ surveys. 

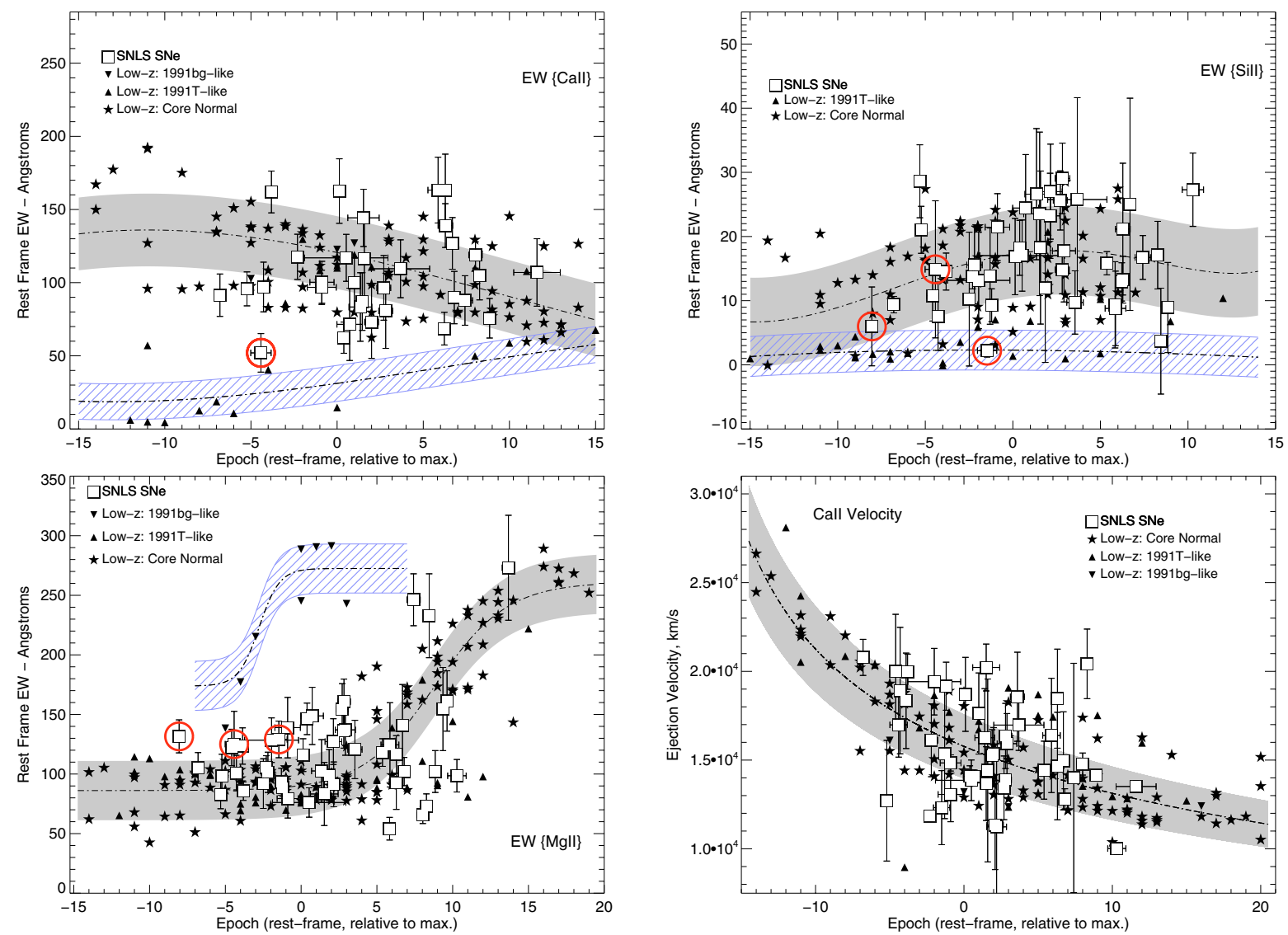

Fig. 6. The results from the SNLS high- $z \mathrm{SNe}$ (open squares) compared to the $E W$ results from the low- $z$ sample (filled symbols). The contours in each plot are the same as Fig. 4. The objects that were identified as 1991T-like SNe are circled in red.

Table 4. A quantitative comparison of the spectroscopic features in high-z supernovae (as measured with $E W$ and CaII $H \& \mathrm{~K}$ ejection velocities) and the mean trends exhibited for these quantities by local $\mathrm{SNe}$ Ia. These results show that there are no significant differences between distant SNLS objects and nearby SNe, with the exception of $E W\{\mathrm{MgII}\}$.

\begin{tabular}{lccc}
\hline \hline Feature & $\begin{array}{c}\text { Reduced }^{a} \\
\chi^{2}\end{array}$ & DOF $^{b}$ & Prob $^{c}$ \\
\hline$E W\{$ CaII $\}$ & 1.10 & 30 & 0.67 \\
$E W\{$ SiII $\}$ & 0.47 & 40 & $<0.002$ \\
$E W\{\mathrm{MgII}\}$ & 1.86 & 44 & $>0.999$ \\
$v_{\mathrm{ej}}$ & 1.03 & 45 & 0.64 \\
\hline
\end{tabular}

${ }^{a} \chi^{2} /$ d.o.f. calculated for the measurement/data set in question and the corresponding low $-z$ mean trend;

${ }^{b}$ Degrees of freedom used when calculating the reduced $\chi^{2}$, $N_{\text {datapoints }}-1$;

${ }^{c}$ Probability $(0.00-1.00)$ that the null hypothesis, that the high- $z$ data are consistent with the trend displayed by low $-z \mathrm{SNe}$, can be rejected.

Overluminous 1991T-like SNe Ia have less absorption from IME at certain epochs. Three outliers in the $E W\{\mathrm{CaII}\}$ and $E W\{\mathrm{SiII}\}$ results have evidence of being similar to the known class of 1991T-like objects. Their spectra are shown in Fig. 7. 03D4cj displayed a smaller amount of SilI than a typical core normal object. The error bars on this measurement are relatively large $(E W\{\mathrm{SiII}\}=5.9 \pm 6.1 \AA)$, but the photometric data for this object supports the conclusion that it is a 1991T-like SN; Astier et al. (2006) stated that the lightcurve from this SN was incompatible with the model used to fit all of the other SNLS SNe.
The $\chi^{2}$ template fits to $03 \mathrm{D} 4 \mathrm{cj}$ also indicate that it is similar to SN 1991T.

The 1991T-like classification also appears valid for SNLS supernova $03 \mathrm{D} 1 \mathrm{~cm}$. This $\mathrm{SN}$ has a very low $E W\{\mathrm{CaII}\}$ measurement and an $E W\{\mathrm{SiII}\}$ result that (within large error bars, $E W\{\mathrm{SiII}\}=14.8 \pm 10.6 \AA$ ) is also consistent with the low values measured in 1991T-like objects. Photometrically, 03D1 cm is also more like a 1991T-like SN as it had one of the highest stretch values from the entire set of Gemini SNLS data at $s=1.173 \pm 0.061$ (Astier et al. 2006). The best matching template spectrum also corresponds to an overluminous low- $z$ $\mathrm{SNe}$. The spectral and photometric dates do differ (+1 day and -4.42 days, respectively) in this case, but the low $\mathrm{S} / \mathrm{N}$ on this object and inherent uncertainty on spectral dating with this template matching method (which is on the order of a few days) means that this is not necessarily enough evidence to outweigh the 1991T-like $E W$ and $s$ values of 03D1 cm.

The SNLS SN 04D3mk also had a very low, well constrained $E W\{\mathrm{SiII}\}$ value. At the epoch this object was observed, the $E W\{\mathrm{MgII}\}$ result cannot differentiate between normal and overluminous $\mathrm{SNe}$ and the $E W\{\mathrm{CaII}\}$ measurement was unusable as it fell on the gap between GMOS detectors (e.g., Sect. 3.2). The final photometry of this object did not reveal any photometric peculiarities, but the $\chi^{2}$ template matches to $04 \mathrm{D} 3 \mathrm{mk}$ singled out overluminous SNe Ia spectra only as the most similar low- $z$ objects. This supports the quantitative similarities of 04D3mk to this particular SN Ia sub-type. With this evidence, SNLS objects 03D $1 \mathrm{~cm}$ and 04D3mk are the most distant supernova (at $z=0.87$ and $z=0.812$, respectively) to be shown to be spectroscopically similar to the 1991T-like SNe Ia sub-types. 

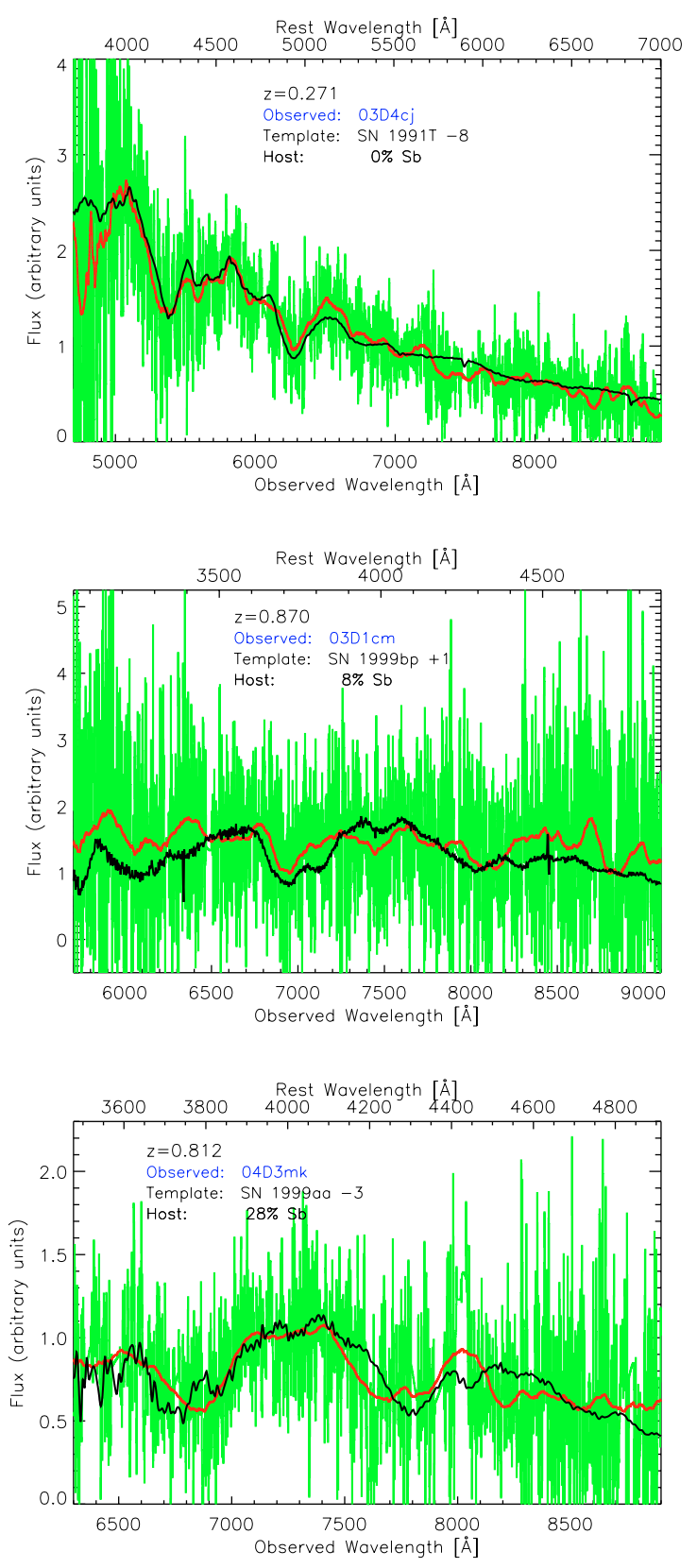

Fig. 7. Three high- $z$ SNe Ia - 03D4cj, 03D1cm and 04D3mk - that demonstrate a quantitative similarity to the 1991T-like SNe Ia sub-type. The green spectrum is the observed spectrum without any binning. The black spectrum is the best-fitting SN from the low- $z$ library and the red spectrum a smoothed version of the observed spectrum. The primary evidence for the similarity of these objects to 1991T-like SNe is the $E W\{\mathrm{CaII}\}$ and $E W\{\mathrm{SiII}\}$ measurements, although in each case the $\chi^{2}$ fits to SN 1991T or SN 1999aa were quantitatively better than those of core normal SNe Ia. Photometric information also supports this subtype identification for these three $\mathrm{SNe}$.

To reiterate, the primary source of this identification for these objects is the $E W$ measurements which are quantitatively more similar to the overluminous sub-class of SNe Ia than other subtypes.

The SNLS SNe 03D1co and 05D3mq are 1991bg-like candidates as their large $E W\{\mathrm{MgII}\}$ values are more consistent with the excess absorption measured in the subluminous low $z$ SNe. However, these two high- $z$ SNe had measurable amounts of SiII, a feature that has yet to be observed in any $1991 \mathrm{bg}$-like $\mathrm{SNe}$ because it is obscured by additional absorption from TiII and FeII. This evidence, combined with the normal photometric parameters (Astier et al. 2006) derived for these objects and the fact that template matches to $1991 \mathrm{bg}$-like objects resulted in poor $\chi^{2}$ results, is enough to justify that these $\mathrm{SNe}$ are more like core normal SNe than any of the underluminous $\mathrm{SNe}$ observed to date.

\subsection{Correlations with host galaxy type}

\subsubsection{Low- $z$ spectra}

The general trend of brighter $\mathrm{SNe}$ and a higher dispersion of $\mathrm{SNe}$ Ia peak magnitudes in late-type galaxies is well documented (Hamuy et al. 1996, 2000; Sullivan et al. 2003). Yet only a few quantitative spectroscopic corollaries (Branch \& van den Bergh 1993; Branch et al. 1996) have been made that agree or disagree with this trend. Kolmogorov-Smirnov (KS) tests were thus used to explore any differences between the spectroscopic parameters measured in SNe from different host types in our low- $z$ sample. This test was carried out on the residuals of these measurements, where the residual for each $E W$ and $v_{\mathrm{ej}}$ value is defined as the difference between the measurement and the corresponding low$z$ mean trend.

The host type for each low $z \mathrm{SN}$ was grouped into the broad categories of "E/S0" and "Spiral" ( $\mathrm{Sa}, \mathrm{Sb}, \mathrm{Sc}$ and irregular) galaxies. The published morphology (from the Nearby Galaxy Catalogue and other appropriate databases) of each SN host galaxy (based on the hosts listed in the SNe reference papers, see Table 1) was used to determine which group was appropriate for each SN host.

A comparison of the residuals for the four measurements discussed in this paper, grouped according to these two broad host galaxy groups, are displayed in Fig. 8. At low- $z$ the only feature that shows a significant trend with host type is SiII.

\subsubsection{High-z spectra}

Another test for possible evolution is to determine whether the correlations between low- $z \mathrm{SNe}$ Ia characteristics and host morphology are observed within the high- $z$ set. The host types were estimated for the SNLS objects that had clear host galaxy features in their spectra and placed into one of three broad categories; "E/S0" for early (elliptical and S0 hosts), "Early Spirals" for objects with spectroscopic features similar to $\mathrm{Sa}$ and $\mathrm{Sb}$ galaxies, and "Late Spirals" for Sc and irregular hosts. The sub-set of SNLS SNe Ia with indentifyable host galaxies includes 45 of the 54 SNLS objects that had $E W$ or $v_{\text {ej }}$ results.

The distribution of these SNLS measurements, displayed in the histograms in the bottom of Fig. 8, do show clear similarities to the low $-z$ distributions (top, Fig. 8). The distant late type galaxies host $\mathrm{SNe}$ that display a smaller amount of SiII than objects from early hosts and the ejection velocities measured in spiral galaxies peak at a higher value. A comparison of the mean values for each measurement (again after subtracting the low- $z$ trend to remove dependence on $\mathrm{SN}$ phase) divided by host type supports this conclusion, as can be seen from Table 5 .

Similar to the low- $z$ results, the high $-z$ data show a trend with regards to host type and $E W\{\mathrm{SiII}\}$, though at a slightly lower significance because of the larger high- $z$ uncertainties. We also see a possible difference in MgII that is not seen at low $-z$. Thus, the KS tests of the distribution of high- $z E W\{\mathrm{SiII}\}$ and $v_{\mathrm{ej}}$ results do not show as significant of a statistical difference between $\mathrm{SNe}$ from different host types. This is probably due to the smaller 

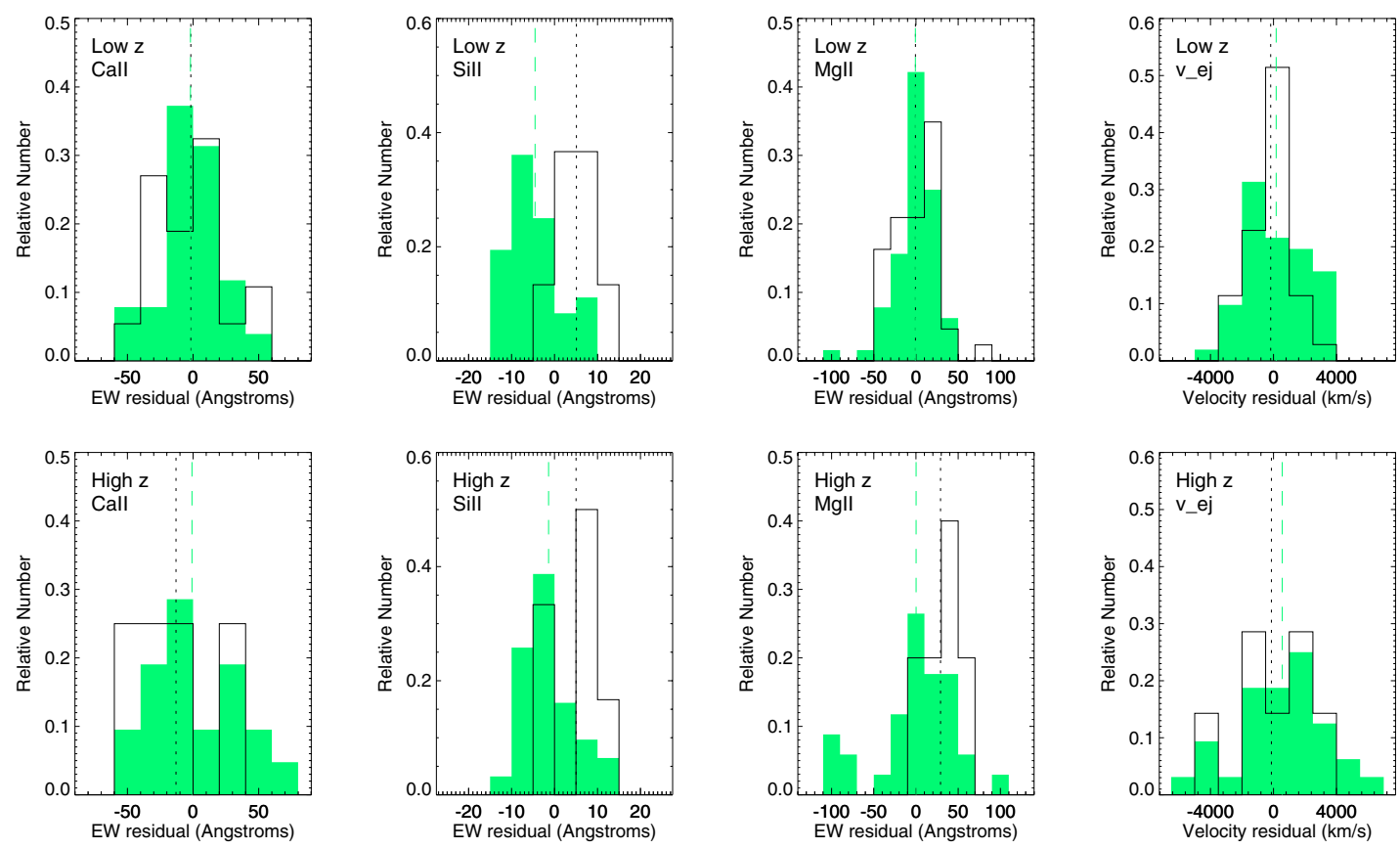

Fig. 8. Histograms comparing the residuals of $E W$ and CaII $v_{\mathrm{ej}}$ measurements in SNe from E/S0 (open histograms) and spiral (filled histograms) host galaxies. The residuals from low- $z$ SNe are displayed in the top row and the SNLS high- $z$ results are on the bottom. The vertical dashed line in each panel is the mean value for each distribution. The histograms have been normalized to have the same area. The distribution of low- $z$ SNe results show a significant difference for the objects from early and late hosts (as determined with KS tests). The high- $z E W\{\mathrm{SiII}\}$ results show a similar trend while $E W\{\mathrm{MgII}\}$ distributions display a difference that is not seen at low- $z$.

Table 5. Comparison of SNe Ia spectral features in different host types at low and high- $z$. The values given are the mean and error in the mean of the residuals from the low- $z$ trend.

\begin{tabular}{lcc}
\hline \hline Feature & \multicolumn{2}{c}{ SNe Measurement } \\
& E/S0 & Spiral $z$ \\
\hline Low- $z$ & & \\
\hline$E W\{\mathrm{CaII}\}-\AA$ & $-1.7 \pm 4.7$ & $-2.0 \pm 3.2$ \\
$E W\{\mathrm{SiII}\}-\AA$ & $5.1 \pm 0.7$ & $-4.5 \pm 0.9$ \\
$E W\{\mathrm{MgII}\}-\AA$ & $-0.6 \pm 3.8$ & $-0.7 \pm 2.9$ \\
$v_{\mathrm{ej}}-1000 \mathrm{~km} \mathrm{~s}^{-1}$ & $-0.18 \pm 0.23$ & $0.17 \pm 0.27$ \\
\hline High- $z$ & & \\
\hline$E W\{\mathrm{CaII}\}-\AA$ & $-13.0 \pm 16.0$ & $-0.8 \pm 7.2$ \\
$E W\{\mathrm{SiII}\}-\AA$ & $5.0 \pm 2.4$ & $-1.4 \pm 1.1$ \\
$E W\{\mathrm{MgII}\}-\AA$ & $29.1 \pm 9.5$ & $0.1 \pm 7.7$ \\
$v_{\mathrm{ej}}-1000 \mathrm{~km} \mathrm{~s}^{-1}$ & $-0.13 \pm 0.90$ & $0.55 \pm 0.50$ \\
\hline
\end{tabular}

number of measurements in the high- $z$ data in addition to the larger errors. For example, the measured maximum deviation between the $E W\{\mathrm{SiII}\}$ distribution in the high- $z$ galaxies (KS statistic $D$ ) is $D=0.50$. This value is comparable to the low- $z D=0.64$ and would indicate a significant difference if the high- $z$ sample was larger by only 10 measurements.

The trend for lower $E W \mathrm{~s}$ and higher ejection velocities in late type galaxies seen at low and now also high- $z$ is a corrolary to the broad-band photometric study of SNLS SNe host galaxies completed by Sullivan et al. (2006b), which showed that the lightcurve shapes of high- $z \mathrm{SNe}$ demonstrate a dependence on host galaxy star formation rates in a manner consistent with low$z$ objects.

\subsubsection{Dependence on redshift}

The high- $z E W$ and $v_{\mathrm{ej}}$ measurements (after subtracting the low- $z$ trend to remove the dependence on SN phase) are plotted against redshift in the left-hand column in Fig. 9. No dependence on redshift is seen. Calculations of Spearman's $\rho$ rank correlation and Kolmogorov-Smirnov tests attest that there are no trends in the data with redshift that are significantly different from random scatter. Thus we see no evidence in our sample for systematic evolution of SNe Ia properties in the large redshift range covered by the SNLS.

On the right side of Fig. 9, the measurements are divided by host galaxy type. The evolutionary histories of host galaxies within a given morphological category have more in common than galaxies of different classfications. The comparison of $\mathrm{SNe}$ Ia spectroscopic features at different redshifts but within similar host types should thus be more sensitive to any evolutionary effects than tests using the bulk properties of the high- $z$ sample as a whole. The panels to the right in Fig. 9 illustrate that there are no redshift-dependent trends within the $\mathrm{SNe}$ results from each host type.

\subsection{EW\{Sill\} - a new SNe la spectroscopic sequence}

During the course of this study, an interesting correlation was found between $E W\{$ SiII $\}$ measurements and $M_{B}$ for the low- $z$ SNe. Figure 10 shows the low- $z$ SNe $E W\{$ SiII $\}$ measurements, taken as close as possible to maximum light (within \pm 7.0 days), compared to the absolute peak $\left(B^{\prime}\right)$ magnitudes (uncorrected for any lightcurve shape-luminosity relationship) that were estimated for 13 of the low- $z$ SNe (see Sect. 2.2.2). A Spearman rank correlation test found a correlation with $3 \sigma$ significance between the measured $E W\{\mathrm{SiII}\}$ and calculated peak magnitude, $M_{B}$. The 
Table 7. SNLS identifications and derived properties for SNe candidates observed from Nov. 2004 to May 2006.

\begin{tabular}{|c|c|c|c|c|c|}
\hline SN & $z$ & $\sigma_{z}^{a}$ & CI \& Type $^{b}$ & Template $^{c}$ & $z$ from $^{d}$ \\
\hline 04D1pu & 0.639 & 0.001 & 3 - SN Ia* & 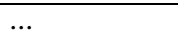 & OII \\
\hline 04D2mh & 0.59 & 0.01 & 3 - SN Ia* & $1996 X+2$ & $\mathrm{SN}$ \\
\hline 04D2mj & 0.513 & 0.001 & 5 - SN Ia & $1992 \mathrm{~A}-1$ & OII, $\mathrm{H} \beta$, OIII \\
\hline 05D1az & 0.842 & 0.001 & 5 - SN Ia & $1996 X+2$ & possible OII \\
\hline 05D1by & 0.298 & 0.001 & 5 - SN Ia & 2002bo -1 & $\mathrm{H} \alpha, \mathrm{H} \beta, \mathrm{OIII}, \mathrm{SII}$ \\
\hline 05D1bz & & & 2 - SN & & \\
\hline 05D1cc & 0.564 & 0.001 & 5 - SN Ia & $1996 X+7$ & $\mathrm{H} \beta$, OII, OIII \\
\hline 05Dlee & 0.558 & 0.001 & 4 - SN Ia & $1992 A+6$ & $\mathrm{H} \& \mathrm{~K}$ \\
\hline 05D1ej & 0.312 & 0.001 & 4 - SN Ia & 1989B -5 & NII \\
\hline 05D1em & 0.866 & 0.001 & 5 - SN Ia & 1999 ee +5 & nearby galaxy \\
\hline 05D1eo & 0.737 & 0.001 & $2-\mathrm{SN}$ & $\ldots$ & H\&K, OII \\
\hline 05D1er & 0.85 & 0.01 & 3 - SN Ia* & 1999aa -1 & SN \\
\hline 05D1hn & 0.149 & 0.001 & 5 - SN Ia & $2003 \mathrm{du}-7$ & $\mathrm{H} \alpha$, SII, OII \\
\hline 05D1ju & 0.707 & 0.001 & 3 - SN Ia* & 1996X +7 & OII, OIII \\
\hline 05D $1 \mathrm{ka}$ & 0.857 & 0.001 & $2-\mathrm{SN}$ & $\ldots$ & $\mathrm{H} \& \mathrm{~K}$ \\
\hline 05D1kl & 0.560 & 0.001 & 3 - SN Ia* & $1990 \mathrm{~N}-7$ & OII, OII \\
\hline 05D2ab & 0.323 & 0.001 & 5 - SN Ia & 1998bu -4 & $\mathrm{H} \alpha$ \\
\hline 05D2ah & 0.184 & 0.001 & 5 - SN Ia & 1994D -5 & $\mathrm{H} \alpha$, SII \\
\hline 05D2ck & 0.698 & 0.001 & 5 - SN Ia & $1992 A+6$ & $\mathrm{H} \& \mathrm{~K}$ \\
\hline 05D2ja & 0.303 & 0.001 & 5 - SN Ia & 1999aw +16 & H $\alpha$, SII, OIII \\
\hline 05D2nt & 0.757 & 0.001 & 4 - SN Ia & 1999ee +3 & OII,H $\beta$, OIII \\
\hline 05D2ob & 0.924 & 0.001 & 4 - SN Ia & 1989B -1 & OII \\
\hline 05D3ax & 0.643 & 0.001 & 5 - SN Ia & 1999aw +12 & OII, OIII \\
\hline 05D3bt & 0.462 & 0.001 & $2-\mathrm{SN}$ & $\ldots$ & $\mathrm{H} \beta, \mathrm{OII}, \mathrm{OIII}$ \\
\hline 05D3cf & 0.419 & 0.001 & 4 - SN Ia & $1999 \mathrm{be}+19$ & $\mathrm{H} \beta$, OII, OIII \\
\hline 05D3ci & 0.515 & 0.001 & 5 - SN Ia & $1992 \mathrm{~A}-5$ & OII, H\&K \\
\hline $05 \mathrm{D} 3 \mathrm{cq}$ & 0.890 & 0.001 & 3 - SN Ia* & 1999ao +5 & $\mathrm{H} \beta$, OII \\
\hline 05D3cx & 0.805 & 0.001 & 4 - SN Ia & $1999 \mathrm{bk}+7$ & possible OII \\
\hline 05D3jq & 0.579 & 0.001 & 4 - SN Ia & $2000 \mathrm{E}+5$ & $\mathrm{H} \beta, \mathrm{H} \gamma, \mathrm{OII}$ \\
\hline 05D3ki & 0.965 & 0.001 & $2-\mathrm{SN}$ & $\ldots$ & OII \\
\hline $05 \mathrm{D} 3 \mathrm{~km}$ & 0.97 & 0.01 & 3 - SN Ia* & 1999bo +1 & SN \\
\hline 05D3kp & $\ldots$ & $\ldots$ & $2-\mathrm{SN}$ & $\ldots$ & $\ldots$ \\
\hline 05D3kt & 0.648 & 0.001 & 5 - SN Ia & 1999ee -2 & $\mathrm{H} \beta$, OII, OIII \\
\hline 05D3lb & 0.647 & 0.001 & 5 - SN Ia & 1999ee 0 & $\mathrm{H} \beta$, OII, OIII \\
\hline 05D3lc & 0.461 & 0.001 & 3 - SN & $\ldots$ & $\mathrm{H} \& \mathrm{~K}$ \\
\hline 05D31w & 0.601 & 0.001 & $2-\mathrm{SN}$ & $\ldots$ & OII \\
\hline 05D3mn & 0.760 & 0.001 & 3 - SN Ia* & $1999 \mathrm{av}+2$ & possible OII \\
\hline 05D3mh & 0.67 & 0.01 & 4 - SN Ia & 1998aq -8 & SN \\
\hline 05D3mq & 0.246 & 0.001 & 5 - SN Ia & $1992 A+9$ & $\mathrm{H} \alpha$ \\
\hline 05D3mx & 0.46 & 0.01 & 4 - SN Ia & 1998bu +8 & SN \\
\hline 05D3ne & 0.169 & 0.001 & 5 - SN Ia & 1994D -4 & SDSS host spectrum \\
\hline 05D4av & 0.509 & 0.001 & 3 - SN Ia* & 2002 bo -4 & OII \\
\hline 05D4bm & 0.375 & 0.001 & 5 - SN Ia & $1996 X-1$ & $\mathrm{H} \beta$, OII, OIII \\
\hline 05D4ca & 0.607 & 0.001 & $2-\mathrm{SN}$ & $1988 A+3$ & $\mathrm{H} \beta$, OII, OIII \\
\hline 05D4cn & 0.763 & 0.001 & 3 - SN Ia* & 2002 bo -1 & OII, H\&K \\
\hline 05D4dt & 0.407 & 0.001 & 4 - SN Ia & 1994D -2 & $\mathrm{H} \alpha, \mathrm{OII}, \mathrm{H} \& \mathrm{~K}, \mathrm{H} \beta, \mathrm{OIII}$ \\
\hline 05D4dx & 0.793 & 0.001 & 3 - SN Ia* & $1002 \mathrm{~A}+5$ & $\mathrm{H} \& \mathrm{~K}, \mathrm{H} \delta$ \\
\hline 05D4dy & 0.79 & 0.01 & 4 - SN Ia & $1994 \mathrm{D}+2$ & SN \\
\hline 05D4fn & 0.4 & 0.1 & 2 - SN & $\ldots$ & SN \\
\hline 05D4fo & 0.373 & 0.001 & 5 - SN & 1994D -2 & $\mathrm{H} \alpha, \mathrm{H} \& \mathrm{~K}$ \\
\hline 05D4gw & 0.808 & 0.001 & 5 - SN Ia & $1999 b p-2$ & OII \\
\hline 05D4hn & 0.842 & 0.001 & 4 - SN Ia & 1981B 0 & OII, OIII \\
\hline 06D3bz & 0.72 & 0.01 & 4 - SN Ia & 1989B -1 & $\mathrm{SN}$ \\
\hline 06D3cb & $\ldots$ & $\ldots$ & $2-\mathrm{SN}$ & $\ldots$ & $\ldots$ \\
\hline 06D3cc & 0.90 & 0.01 & 3 - SN Ia* & $1990 \mathrm{~N}-7$ & $\ldots$ \\
\hline 06D3cl & 0.555 & 0.001 & 2 - SN & $\ldots$ & OII, OIII, $\mathrm{H} \beta$ \\
\hline 06D3cn & 0.818 & 0.001 & 5 - SN Ia & $1996 X-1$ & OII, OIII, $\mathrm{H} \beta$ \\
\hline 06D3du & 1.0 & 0.1 & $2-\mathrm{SN}$ & $\ldots$ & SN \\
\hline $06 \mathrm{D} 3 \mathrm{dz}$ & 0.294 & 0.001 & $2-\mathrm{SN}$ & $\ldots$ & $\mathrm{H} \alpha$, SII \\
\hline
\end{tabular}

${ }^{a}$ Redshifts identified with host galaxy features are accurate to $\approx \pm 0.001$; redshifts identified;

with template matches are accurate to $\approx \pm 0.01$;

${ }^{b}$ Final object identification and confidence index;

${ }^{c}$ Best low- $z$ spectral fit (name and days past max.);

${ }^{d}$ The host features used to estimate $z$. 

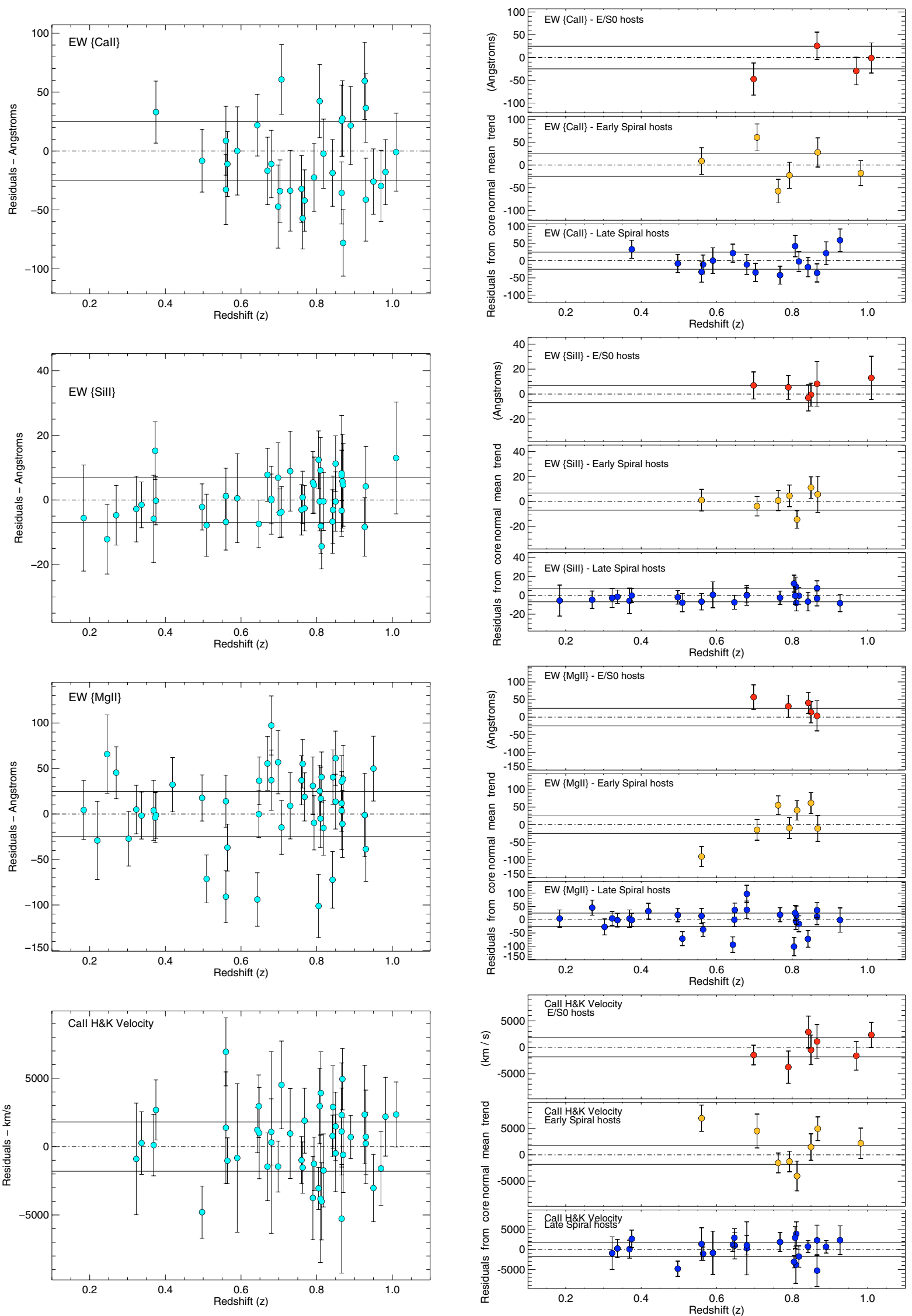

Fig. 9. Left: The high- $z$ residuals (i.e. the difference from low- $z$ SNe Ia core normal mean trend) from the $E W$ and $v_{\text {ej }}$ measurements are plotted against redshift. Right: the same data divided by host galaxy type. 


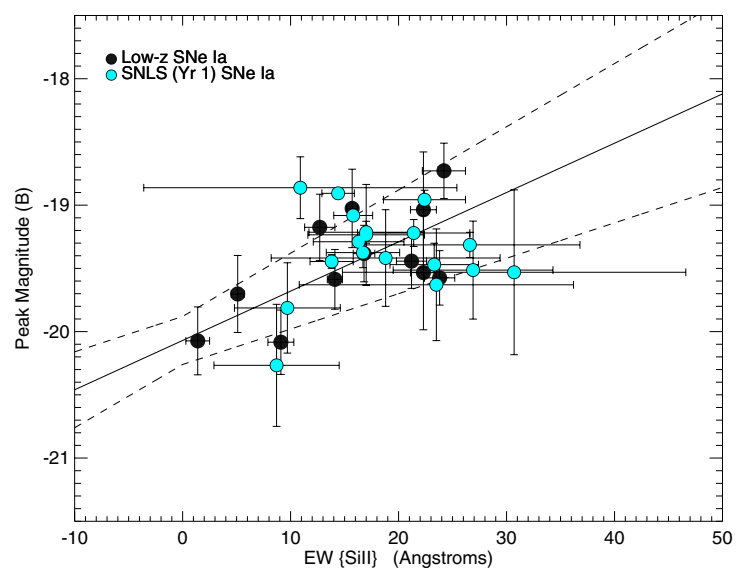

Fig. 10. A comparison of the $E W$ measurement of the "SiII 4000" feature to the absolute $B^{\prime}$ peak magnitudes of low- $z$ SNe Ia (black circles) and distant $\mathrm{SNe}$ (lighter circles). The significant correlation in the low- $z$ data reveals a new spectroscopic sequence in SNe Ia that can be used to estimate $\mathrm{SNe}$ Ia peak magnitudes. The high- $z$ points have larger error bars with regards to both $E W$ and magnitude, but are still consistent with the low $-z$ trend (illustrated with the solid line).

best fit linear correlation of these quantities is described by the following:

$M_{B}=(-20.07 \pm 0.19)+(0.039 \pm 0.011) \times(E W\{\operatorname{SiII}\})$.

The small residuals from this fit place this spectral indicator on par with others in the literature (Wells et al. 1994; Bongard et al. 2006; Nugent et al. 1995a; Hachinger et al. 2006). This spectroscopic sequence is also relatively free of the epoch constraints on similar sequences (Nugent et al. 1995a; Benetti et al. 2005; F04) because $E W\{$ SiII $\}$ evolves little near maximum light and only one measurement is needed to constrain $M_{B}$ with this correlation. Any single $E W$ measurement near this date (the authors found that \pm 7.0 days was best as the $E W\{$ SiII $\}$ values evolved little over this period) yielded the similar results. The wavelength of this feature also makes it ideal as it falls within the optical spectrum out to redshifts of about 1.2 (unlike measurements in the redder end of the spectrum such as the $\lambda 5972$ feature studied in Hachinger et al. 2006).

One major physical parameter that underlies this sequence is temperature (Nugent et al. 1995a; Branch et al. 2006; Baron et al. 2006). For an assumed Chandrasekhar mass $\left(M_{\mathrm{CH}}\right) \mathrm{WD}$ explosion scenario, the distribution of peak magnitudes is based primarily on the different amounts of ${ }^{56} \mathrm{Ni}$ synthesised. Higher production of this isotope, which is made at the expense of IME such as SiII, results in a brighter $\mathrm{SNe}$ and causes the ejecta to be hotter. This tends to overionise the already smaller amount of IME observed at maximum light thus results in the appearance of a smaller amount of SiII in the spectra of hotter and brighter $\mathrm{SNe}$ at these epochs.

Evidence that this spectroscopic sequence is similar at high- $z$ is illustrated in the SNLS points in Fig. 10. In this figure the absolute magnitudes of the SNLS high-z SNe, uncorrected for lightcurve stretch or colour adjustments, were computed assuming the best fit cosmology and published $m_{B}$ values from Astier et al. (2006).

Within the relatively large $E W$ error bars, the SNLS supernovae are still consistent with the low $-z$ trends in this parameter space. The next logical step is to determine whether the application of this relation to high- $z \mathrm{SNe}$, just like the use of $s$ or $\Delta_{m_{15}}$ parameters, decreases the scatter of luminosity distances in a Hubble diagram. This test is displayed in Fig. 11.
For this test, the set of SNLS SNe that were published in the first year SNLS cosmology results and had measured $E W\{\mathrm{SiII}\}$ values were used. A total of $18 \mathrm{SNe}$ Ia from Astier et al. (2006) met these criteria. The high- $z E W\{$ SiII $\}$ measurements were used with the relation estimated from the low $-z$ sample (Eq. (6)) to estimate the absolute peak $B$-band magnitudes (now termed $M_{\text {SiII }}$ ).

The distance modulus $(\mu)$ for these distant objects was then calculated as

$\mu=m_{B}-M_{\text {SiII }}$

where $m_{B}$ is the observed peak $B^{\prime}$ magnitude previously published in Table 9 of Astier et al. (2006). The uncertainties in the linear $M_{B}-E W\{\mathrm{SiII}\}$ fit and $m_{B}$ were propagated along with the $E W$ errors during this calculation.

Figure 11 indicates that the $E W\{\mathrm{SiII}\}$ sequence does provide a plausible method to use for estimating peak SNe Ia distances for cosmology. The dispersion in the $E W\{$ SiII $\}$-estimated distances about the best-fit cosmological model from Astier et al. (2006) is 0.26 mag., which is near the computed residuals of $0.23 \mathrm{mag}$. from the Astier et al. (2006) stretch and colourcorrected SNLS SNe. Both of these values are much improved over the 0.37 magnitude dispersion from the un-corrected $\mathrm{SNe}$ magnitudes in this sub-set of 18 high- $z$ SNLS SNe. The application of this correlation to $\mathrm{SNe}$ Ia cosmology is further discussed in Bronder (2006).

\section{Discussion}

As discussed in Sect. 4, the spectroscopic measurements and comparisons completed on this new set of distant $\mathrm{SNe}$ have shown that these SNLS-observed objects display an overall similarity with the SNe Ia observed at low redshifts. We find no significant difference between the SNe Ia samples for three of the four features studied and a possible difference in the fourth feature $(E W\{\mathrm{MgII}\})$. We also find similar trends with host type at low and high redshift. Below we discuss these results in the context of the population of progenitors in addition to the rates of various Type Ia sub-types in the SNLS high- $z$ sample.

\subsection{Low-z and high-z discrepancy - EW\{MgII\}}

In Sect. 4.2 a possible difference in $E W\{\mathrm{MgII}\}$ between low and high- $z$ samples was presented. For the reason given in that section (namely the differences in the time-sampling of observations in the two samples), the significance of this difference may not be high. This was also sugested in a similar study by Garavini et al. (2007), who also noted the need for an analysis of a larger sample of normal SNe Ia to more accurately determine the true mean trend for this measurement.

However, it is interesting to consider possible physical explanations, for example it is possible that it is a sign of metallicity differences. Explosion and nucleosynthesis calculations from Travaglio et al. (2005) and Roepke et al. (2006), which were computed by modelling thermonuclear burning by deflagration (as opposed to delayed detonation or detonation only) of a $\mathrm{C}+\mathrm{O}$ WD progenitor, showed that Type Ia SNe from higher metallicity $(Z)$ environments produce a significantly lower (by a factor of 10) amount of ${ }^{24} \mathrm{Mg}$. In their results, the abundances of $\mathrm{Si}$ and $\mathrm{Ca}$ were relatively unaffected by metallicity changes. Thus, it may be that the group of high- $z \mathrm{SNe}$ that are below the mean trend for this feature are all SNe from higher metallicity progenitors. The normal $E W\{\mathrm{SiII}\}$ and $E W\{\mathrm{CaII}\}$ measurements in 

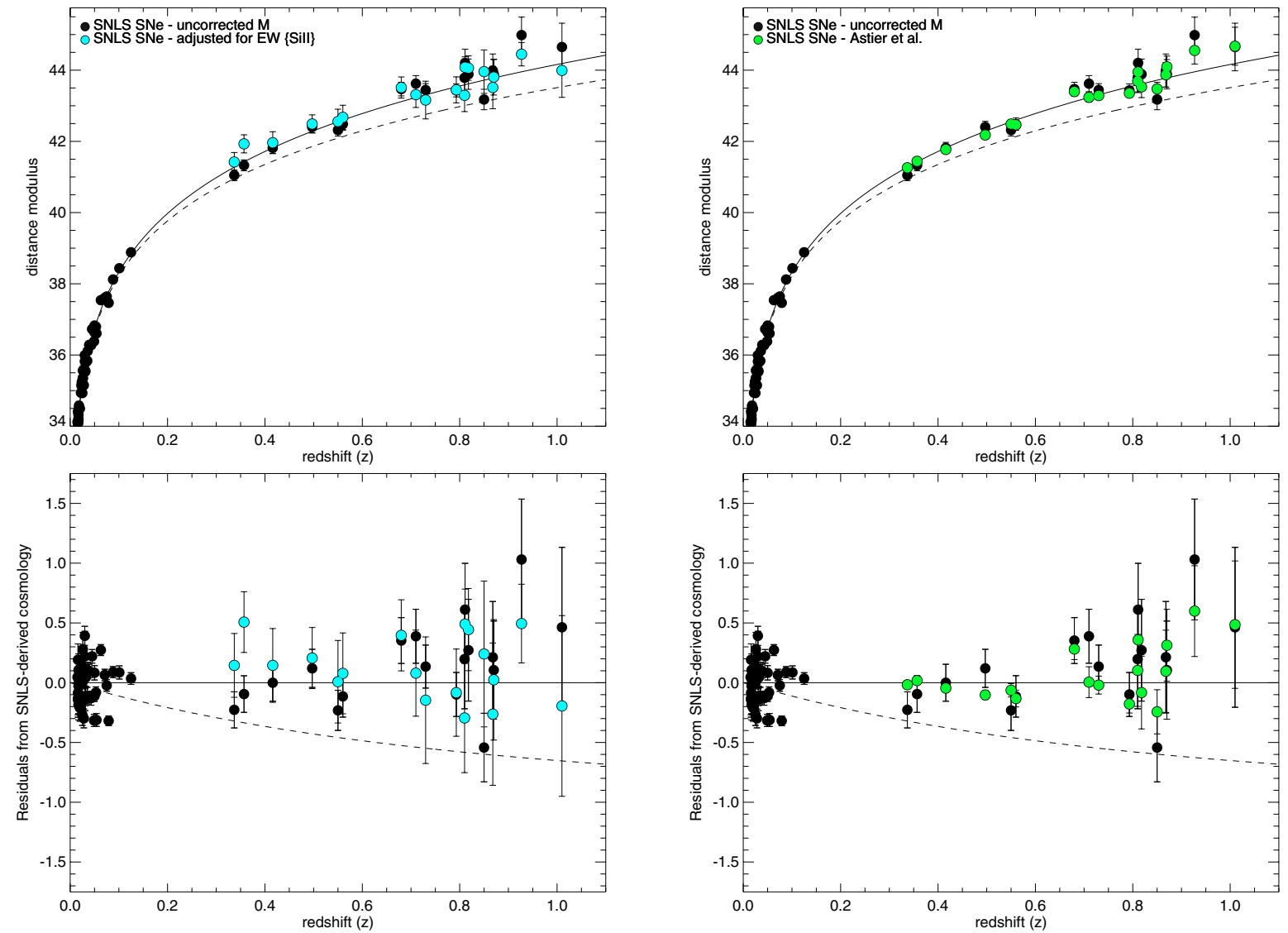

Fig. 11. Hubble diagrams for a subset of SNe Ia from the SNLS first year dataset. Solid points show the distance moduli uncorrected for stretch or colour effects. Left: the lighter (or coloured) points show distance moduli that were calculated with peak magnitudes estimated by the $E W\{$ SiII $\}$ sequence. Right: the lighter (or coloured) points show distance moduli that were corrected with $s$ (stretch) and $c$ (colour) corrections (Astier et al. 2006). The solid line indicates the best fit cosmology from the first year SNLS results - a spatially flat Universe with $\Omega_{M}=0.263$ and $\Omega_{\Lambda}=0.737$. The dashed line is the model from a flat Universe with $\Omega_{M}=1.00$. The residuals about this cosmology are shown in the bottom two panels; the uncorrected high- $z$ SNe points have a dispersion about this cosmology of 0.37 magnitudes, while the $E W\{$ SiII $\}$ points have a dispersion of 0.26 magnitudes; this is comparable to the 0.23 mag dispersion in the Astier et al. (2006) results.

these SNe support this explanation within the context of these models.

Conversely, model SNe Ia spectra from progenitors of varying $Z$ calculated by Lentz et al. (2000) showed the opposite effect in the $E W\{\mathrm{MgII}\}$ measurements. The Lentz et al. (2000) spectra were calculated with a similar deflagration model SN (W7 from Nomoto et al. 1984), with the metallicity changes approximated by scaling the number abundances of elements heavier than $\mathrm{O}$ in the unburned $\mathrm{C}+\mathrm{O}$ layer. The final IME abundances were not published in Lentz et al. (2000), so they could not be compared directly to the Travaglio et al. (2005) results. Instead, the $E W$ measurements defined for this study were made directly on the final Lentz et al. (2000) model spectra. These measurements showed that the SNe with higher $Z$ had larger $E W\{\mathrm{MgII}\}$ measurements at pre-maximum epochs but then evolved such that the different $Z$ models were nearly indistinguishable after maximum light.

Another factor that may be contributing to this difference is the trend for the SNLS to find more slightly bluer, brighter objects (Astier et al. 2006). F04 noted that the evolution of different $\mathrm{SNe}$ along the mean trend for $E W\{\mathrm{MgII}\}$ correlated with luminosity, with brighter objects making the "jump" to higher $E W$ at later times. The small group of outliers below the $E W\{\mathrm{MgII}\}$ mean trend between +5 and +10 days may just be reflecting a slightly brighter group of SNe in the high- $z$ sample. The average $s$ value within these $\mathrm{SNe}$ is just above 1.0 (this corresponds to brighter SNe, see Fig. 9 in Astier et al. 2006), which supports this hypothesis. This feature comparison may be more sensitive to slight differences in the strecth distribution of the samples than the other $E W$ results. Further investigation into this parameter, both observationally and theoretically, is certainly warranted.

\subsection{Rate of peculiar $\mathrm{SNe}$}

The $E W$ results of this large high- $z$ set note 2 to 3 objects (out of 54 distant supernovae with $E W$ results, $\sim 3-6 \%$ ) that are spectroscopically similar to the class of 1991T-like SNe sub-types that have been observed locally. Previous studies of distant $\mathrm{SNe}$ Ia have noted a similarly small percentage of these sub-types in the high- $z$ samples; only spectra from SNe 2002iv $(z=0.231)$, 2003jn $(z=0.33), 2002 \mathrm{fd}(z=0.27)$ (Garavini et al. 2007), and 2003lh $(z=0.56)$ (Matheson et al. 2005; Krisciunas et al. 2005 ) have been shown to be quantitatively similar to $1991 \mathrm{~T}$ or 1999aa. This is in contrast to the apparent contribution of these sub-types to the low- $z$ SNe sample, which is roughly $10 \%$ to $20 \%$ (Li et al. 2001a; Strolger et al. 2002). This has been interpreted as a possible sign of evolution (Branch 2001; Li et al. $2001 \mathrm{a}, \mathrm{b})$ or of a significant difference between the nearby and distant $\mathrm{SNe}$ Ia populations. The identification of the 3 possible 
1991T-like objects in this high-z sample nearly doubles the number of these sub-types observed at higher redshifts, but the percentage of identified 1991T-like SNe is still much lower than at low- $z$. It is difficult to determine the significance of this apparent discrepancy due to the unknown influence of selection effects. For example, the low- $z$ peculiar objects are often singled out for spectroscopic follow up and thus may be over-represented in the nearby sample. Another complication is the uncertainty with regards to whether the overluminous 1991T-like and 1999aa-like $\mathrm{SNe}$ are distinct sub-classes of Type Ia SNe or if they represent a possible continuum of thermonuclear SNe (Branch et al. 2006). Note that the $E W$ and $v_{\text {ej }}$ measurements cannot readily address this latter issue; the overluminous high- $z$ objects are referred to as "1991T-like" in the interest of brevity only and does not represent an attempt to clarify the debate on the 1991T-1999aa relationship.

It may be that the difference between the fraction of these sub-types at different redshifts is primarily an effect of the epoch coverage differences between these data sets. SNe 1991T and 1999aa displayed unusual spectroscopic features primarily before maximum light and then evolved to look little different than core normal SNe. Within the low- $z$ set considered here, $77 \%$ of the $\mathrm{SNe}$ were observed at least once prior to maximum light, compared to only $33 \%$ ( 18 of $54 \mathrm{SNe}$ ) of the high- $z$ sample. With the simple assumption that this factor of $\sim 2$ difference has reduced the number of overluminous SNe possibly identified in the high- $z$ sample by a similar amount, the disparity in the percentage of overluminous sub-type identifications between the two data sets is explained.

Even if this explanation is true, there is still a lack of of the underluminous $1991 \mathrm{bg}$-like $\mathrm{SNe}$ at high redshifts. This is perhaps expected for this data set as many factors work against SNLS spectroscopic identification of these objects. Mostly, it is because they are intrinsically much fainter and thus harder to observe photometrically and spectroscopically. This implies that even if the SNLS is observing these objects spectroscopically, they would be much harder to conclusively type. Thus the lack of $1991 \mathrm{bg}$-like SNe Ia in this set is not unexpected.

The end of Sect. 2.1.1 summarizes that, due to the SNLS survey setup, high- $z$ SNe with vastly different properties than the nearby objects would most likely not be selected for spectroscopic observation. Thus, the conclusions of the tests made here cannot rule out that such a population of SNe Ia exists at high- $z$, although none of the results show any indication of new sub-classes of distant SNe. However, we note that occasionally very rare and unsual objects are found (see SNLS object 03D3bb in Howell et al. 2006, for example).

\subsection{Implications for progenitors}

Comparisons of SNe Ia across different host galaxy types can also reveal keys to the progenitor scenarios for these objects. One model of SNe Ia formation (Scannapieco \& Bildsten 2005) has used the implications of observed SNe Ia environmental dependencies (Wang et al. 1997; Umeda et al. 1999; Hamuy et al. 2000; Howell 2001; Mannucci et al. 2005) to create a model that describes SNe Ia as two distinct populations; a "prompt" component dependent on the galaxy star formation rate and a "delayed" component whose rate is proportional to galaxy mass. This model has been supported with SNLS data (Sullivan et al. 2006b) and may be useful in predicting any evolutionary shifts in Sne Ia properties (Howell et al. in prep.). The measurements made in this study can also be put into the context of this "twocomponent" model. Table 5 and Fig. 8 show a trend of SilI with host type (at both low and high-z) in the direction expected from this two-component model. At all redshifts, $\mathrm{SNe}$ in spiral hosts show weaker SiII. Assuming that these early type galaxies are star-forming, and hence host the "prompt" SNe Ia (which also tend to be the brighter, higher stretch $\mathrm{SNe}$ Ia), and that the $E W\{\mathrm{SiII}\}-M_{B}$ correlation is valid, then the trend of lower $E W\{\mathrm{SiII}\}$ measurements in $\mathrm{SNe}$ from late type galaxies agrees with the main aspects of the two-component model.

Another implication of this model is that as observations are extended to higher redshifts, a larger sampling of SNe Ia from the "prompt" star formation-based population would be expected. As these "prompt" SNe are brighter than their "delayed" counterparts, this should correspond to additional observations of SNe with high $s$ values and correspondingly low $E W\{\mathrm{SiII}\}$ measurements. This effect can be tested in the SNLS sample considered here by combining the high- $z$ results from all galaxy types. $\chi^{2}$ tests and measures of correlation show no statistical difference in $E W\{\mathrm{SiII}\}$ between low and high- $z$ samples (see also Fig. 9). However, this could be due to a lack of sensitivity to detect this subtle drift in the population. A possible shift of $\approx 10 \%$ in the brightness (or stretch) of the high- $z$ population (Howell et al. in prep.) would correspond to a change of the order of a few $(\approx 4) \AA$ in $E W\{$ SiII $\}$; Table 5 shows that the error in the means of the distribution of high- $z E W\{\mathrm{SiII}\}$ is on the order of this shift and is much higher than the corresponding error in the low- $z$ results. An SNLS sample with 2-3 times more SNe (which will be available before the survey is complete) should be able to detect this shift toward the "prompt" SNe.

\section{Conclusions}

New results from a quantitative study of a large set of spectra from high- $z$ SNe Ia used for cosmological fitting have been compared to corresponding measurements on a sample of SNe Ia observed locally. The measurements from all of the studied high- $z$ spectra are within the range of the results at low- $z$. A statistical comparison of these data sets reveals that there is no significant difference (less than $2 \sigma$ ) in the strength of 2 SNe Ia-specific absorption features and the ejection velocity of $\mathrm{CaII} H \& \mathrm{~K}$. The 4th measurement - which quantifies absorption from $\mathrm{MgII}$ and other ions near $4300 \AA$ - shows a possible difference that should be investigated further.

These methods also reveal a new spectroscopic sequence in SNe Ia based on the strength of the SiII feature near $4000 \AA$ at epochs close to maximum luminosity. An initial test of this sequence was made on a sub-set of 18 SNLS SNe that had the appropriate $E W\{\mathrm{SiII}\}$ measurement and were published with previous SNLS cosmology results (Astier et al. 2006). This demonstrated that the $E W\{\mathrm{SiII}\}-M_{B_{\text {peak }}}$ relation reduces the scatter in high- $z$ luminosity distance estimates in a manner consistent with the lightcurve shape-luminosity corrections that are currently used to standardise SNe Ia peak magnitudes for cosmology.

At present this type of spectroscopic analysis is one of the most promising avenues of investigating the dispersion, homogeneity and possible evolution of distant Type Ia supernovae. This paper has used empirical techniques on the largest set of distant SNe Ia data studied to date. Although the selection method used to generate the SNLS high- $z$ sample implies that the existence of a new population of Type Ia SNe at high- $z$ with unusual spectral properties cannot be ruled out, a large set of high- $z \mathrm{SNe}$ have been shown to be spectroscopically similar to nearby objects. At high- $z$ the same trends of SN properties with host type are seen as at low- $z$, and furthermore, within each host 
galaxy class no evidence for evolution in SN properties with redshift is seen. These results represent an important step in addressing one of the most pressing issues for Type Ia cosmology.

Acknowledgements. The SNLS collaboration gratefully acknowledges the assistance of Pierre Martin and the CFHT Queued Service Observations team We also thank the Gemini queue observers and support staff for both taking the data presented in this paper and making observations available quickly. T.J.B. is grateful to Exeter College and the Alberta-Bart Holaday Scholarship. T.J.B. would also like to thank the EU Type Ia SNe RTN "young researchers" - particularly Gabriele Garavini and Gaston Folatelli - for all of their assistance. Canadian collaboration members acknowledge support from NSERC and CIAR; French collaboration members from CNRS/IN2P3, CNRS/INSU and CEA; Portuguese Collaboration members acknowledge support from FCT Fundacao para a Ciencia e Tecnologia; UK collaborators acknowledge support from the Science and Technolgy Facilities Council (STFC, formerly PPARC). SNLS relies on observations with MegaCam, a joint project of CFHT and CEA/DAPNIA, at the Canada-France-Hawaii Telescope which is operated by the National Research Council of Candada, the Institut National des Science de l'Univers of the Centre National de la Recherche Scientifique of France, and the University of Hawaii. This work is based in part on data products produced at the Canadian Astronomy Data Centre as part of the CFHT Legacy Survey, a collaborative project of the National Research Council of Canada and the French Centre national de la recherche scientifique. The Work is also based on observations obtained at the Gemini Observatory, which is operated by the Association of Universities for Research in Astronomy, Inc., under a cooperative agreement with the NSF on behalf of the Gemini partnership: the National Science Foundation (United States), the STFC (United Kingdom) the National Research Council (Canada), CONICYT (Chile), the Australian Research Council (Australia), CNPq (Brazil) and CONICET (Argentina). This research used observations from Gemini program numbers: GN-2005A-Q-11, GN-2005B-Q-7, GN-2006A-Q-7, GS-2005A-Q-11 and GS-2005B-Q-6.

The views expressed in this article are those of the authors and do not reflect the official policy or position of the United States Air Force, Department of Defense, or the US Government.

\section{References}

Allen, S. W., Schmidt, R. W., \& Fabian, A. C. 2002, MNRAS, 334, 11 Altavilla, G., Fiorentino, G., Marconi, M., et al. 2004, MNRAS, 349, 1344 Anupama, G. C., Sahu, D. K., \& Jose, J. 2005, A\&A, 429, 667

Astier, P., Guy, J., Regnault, N., et al. 2006, A\&A, 447, 31

Balland, C., Mouchet, M., Pain, R., et al. 2006, A\&A, 445, 387

Barbon, R., Benetti, S., Capellaro, E., et al. 1990, A\&A, 237, 79

Baron, E., Bongard, S., Branch, D., et al. 2006, ApJ, 645, 480

Barris, J., Tonry, J. L., Blondin, S., et al. 2004, ApJ, 602, 571

Benetti, S., Meikle, P., Stehle, M., et al. 2004, MNRAS, 348, 261

Benetti, S., Cappellaro, E., Mazzali, P. A., et al. 2005, ApJ, 623, 1011

Blondin, S., Dessart, L., Leibundgut, B., et al. 2006, AJ, 131, 1648

Bongard, S., Baron, E., Smadja, G., Branch, D., \& Hauschchildt, P. H. 2006, in prep.

Branch, D. 2001, PASP, 113, 169

Branch, D., \& van den Bergh, S. 1993, AJ, 105, 2231

Branch, D., Lacy, C. H., McCall, M. L., et al. 1983, ApJ, 270, 123

Branch, D., Drucker, W., \& Jeffrey, D. J. 1988, ApJ, 330, L117

Branch, D., Romanishin, W., \& Baron, E. 1996, ApJ, 465, 73

Branch, D., Garnavich, P., Matheson, T., et al. 2003, AJ, 126, 1489

Branch, D., Dang, L. C., Hall, N., et al. 2006, PASP, 118, 560

Bronder, T. J. 2006, Ph.D. Thesis, Oxford University

Candia, P., Krisciunas, K., Suntzeff, N. B., et al. 2003, PASP, 115, 277

Capellaro, E., Patat, F., Mazzali, P. A., et al. 2001, ApJ, 549, 215

Elias-Rosa, N., Benetti, S., Cappellaro, E., et al. 2006, MNRAS, 369, 1880

Filippenko, A. V., Richmond, M. W., Matheson, T., et al. 1992a, ApJ, 384, L15

Filippenko, A. V., Richmond, M. W., Branch, D., et al., 1992b, AJ, 104, 1543

Fisher, A., Branch, D., Hoeflich, P., \& Khokhlov, A. 1995, AJ, 447, L73

Folatelli, G. 2004, Ph.D. Thesis, University of Stockholm

Gallagher, J. S., Garnavich, P. M., Berlind, P., et al. 2005, ApJ, 634, 210

Garavini, G., Folatelli, G., Goobar, A., et al. 2004, AJ, 128, 387

Garavini, G., et al. 2007, A\&A, submitted
Gibson, B. K., Stetson, P. B., Freedman, W. L., et al. 2000, ApJ, 529, 723

Goldhaber, G., Groom, D. E., Kim, A., et al. 2001, ApJ, 558, 359

Gomez, G., \& Lopez, R. 1998, AJ, 115, 1096

Guy, J., Astier, P., Nobili, S., Regnault, N., \& Pain, R. 2005, A\&A, 443, 781

Hachinger, S., Mazzali, P. A., \& Benetti, S. 2006, MNRAS, 370, 299

Hamuy, M., Phillips, M. M., Suntzeff, N. B., et al. 1996, AJ, 112, 2391

Hamuy, M., Trager, S. C., Pinto, P. A., et al. 2000, AJ, 120, 1479

Hatano, K., Branch, D., Lentz, E. J., et al. 2000, ApJ, 543, L49

Hillebrandt, W., \& Niemeyer, J. 2000, ARA\&A, 38, 191

Hoeflich, P., Wheeler, J. C., \& Thielemann, F. K. 1998, ApJ, 495, 617

Hook, I., Jørgensen, I., Allington-Smith, J. R., et al. 2004, PASP, 116, 425

Hook, I., Howell, D. A., Aldering, G., et al. 2005, AJ, 130, 2788

Howell, D. A. 2001, ApJ, 554, L193

Howell, D. A., \& Wang, L. 2002, BAAS, 34, 1256

Howell, D. A., Sullivan, M., Perrett, K., et al. 2005, ApJ, 634, 1190

Howell, D. A., Sullivan, M., Nugent, P. E., et al. 2006, Nature, 443, 308

Hsiao, E., et al. ApJ, in press

Jeffrey, D. J., Leibundgut, B., Kirshner, R. P., et al. 1992, ApJ, 397, 304

Kirshner, R., Jeffery, D., Leibundgut, B., Challis, P. M., \& Sonneborn, G. 1993, ApJ, 415, 589

Knop, R., Aldering, G., Amanullah, R., et al. 2003, ApJ, 598, 102

Krisciunas, K., Garnavich, P. M., Challis, P., et al. 2005, AJ, 130, 2453

Leibundgut, B. 2001, ARA\&A, 39, 67

Lentz, E. J., Baron, E., \& Branch, D. 2000, 530, 966

Levan, A., Nugent, P., Fruchter, A., et al. 2005, ApJ, 624, 880

Li, W. D., Qiu, Y. L., Qiao, Q. Y., et al. 1999, AJ, 117, 2709

Li, W., Filippenko, A. V., \& Riess, A. G. 2001a, ApJ, 546, 719

Li, W., Filippenko, A. V., Treffers, R. R., et al. 2001b, ApJ, 546, 734

Mannucci, F., Della Valle, M., Panagia, N., et al. 2005, A\&A, 433, 807

Matheson, T., Blondin, S., Foley, R. J., et al. 2005, AJ, 129, 2352

Mazzali, P., Lucy, L. B., \& Danziger, I. J. 1993, A\&A, 269, 423

Mazzali, P. A., Danziger, I. J., \& Turatto, M. 1995, A\&A, 297, 509

Mazzali, P., Cappellaro, E., Danziger, I. J., et al. 1998, ApJ, 499, L49

Mazzali, P., Benetti, S., Altavilla, G., et al. 2005, ApJ, 623, 37

Nomoto, K., Thielemann, F.-K., \& Yokoi, K. 1984, ApJ, 286, 644

Nugent, P., Kim, A., \& Perlmutter, S. 2002, PASP, 114, 803

Nugent, P., Phillips, M., Baron, E., et al. 1995, ApJ, 455, L147

Patat, F., Benetti, S., Cappellaro, E., et al. 1996, MNRAS, 278, 111

Perlmutter, S., Aldering, G., Goldhaber, G., et al. 1999, ApJ, 517, 565

Phillips, M. M., Phillips, A. C., Heathcote, S. R., et al. 1987, PASP, 99, 592

Phillips, M. M., Lira, P., Suntzeff, N. B., et al. 1999, AJ, 118, 1766

Reindl, B., Tamman, G. A., Sandage, A., \& Saha, A. 2005, ApJ, 624, 532

Riess, A. G., Press, W. H., \& Kirshner, R. P. 1996, ApJ, 473, 88

Riess, A. G., Filippenko, A. V., Challis, P., et al. 1998, AJ, 116, 1009

Riess, A. G., Strolger, L.-G., Tonry, J., et al. 2004, ApJ, 607, 665

Riess, A. G., Li, W., Stetson, P. B., et al. 2005, ApJ, 627, 579

Ropke, F. K., Gieseler, M., Travaglio, C., \& Hillebrandt, W. 2006, A\&A, 453, 203

Saha, A., Sandage, A., Thim, F., et al., 2001, ApJ, 551, 973

Salvo, M. E., Cappellaro, E., Mazzali, P. A., et al. 2001, MNRAS, 321, 254

Scannapieco, E., \& Bildsten, L. 2005, ApJ, 629, L85

Seljak, U., Makarov, A., McDonald, P., et al. 2005, PhRvD, 71, 3515

Spergel, D. N., et al. 2006, in prep.

Spergel, D. N., Verde, L., Peiris, H. V., et al. 2003, ApJS, 148, 175

Stern, D., van Dokkum, P. G., Nugent, P., et al. 2004, ApJ, 612, 690

Stritzinger, M., Hamuy, M., Suntzeff, N. B., et al. 2002, AJ, 124, 2100

Sullivan, M., Ellis, R. S., Aldering, G., et al. 2003, MNRAS, 340, 1057

Sullivan, M., Howell, D. A., Perrett, K., et al. 2006a, AJ, 131, 960

Sullivan, M., Le Borgne, D., Pritchet, C. J., et al. 2006b, ApJ, 648, 868

Strolger, L. G., Smith, R. C., Suntzeff, N. B., et al. 2002, AJ, 124, 2905

Travaglio, C., Hillebrandt, W., \& Reinecke, M. 2005, A\&A, 443, 1007

Tully, R. B., Nearby Host Galaxies Catalogue (Cambridge, UK: Cambridgue University Press)

Turatto, M., Benetti, S., Cappellaro, E., et al. 1996, MNRAS, 283, 1

Turatto, M., Piemonte, A., Benetti, S., et al. 1998, AJ, 116, 2431

Umeda, H., Nomoto, K., Kobayashi, C., Hachisu, I., \& Kato, M. 1999, ApJ, 522, L43

Wang, L., Hoeflich, P., \& Wheeler, J. C., 1997, ApJ, 483, 29

Wells, L. A., Phillips, M. M., Suntzeff, B., et al. 1994, AJ, 108, 2233

Yamaoka, H., Nomoto, K., Shigeyama, T., \& Thielemann, F. K. 1992, ApJ, 393, L55 


\section{Online Material}


Table 6. Observed properties and instrument settings for SNLS SNe candidates observed from November 2004 to May 2006. The magnitudes and "percentage increase" are estimated from the lightcurves and correspond to the date of spectroscopy.

\begin{tabular}{|c|c|c|c|c|c|c|c|c|c|}
\hline$\overline{\text { SN Name }}$ & RA (2000) & Dec (2000) & UT Date & Exposure (s) & Mode & $\lambda_{\text {cent }}$ & seeing $\left({ }^{\prime \prime}\right)$ & Mag $\left(i^{\prime}\right)$ & $\%_{\text {inc }}$ \\
\hline 04D1pu & $02: 27: 28.437$ & $-04: 44: 41.71$ & $2004-12-12$ & 7200 & $\mathrm{~N} \& \mathrm{~S}$ & 720 & 0.49 & 24.08 & 39 \\
\hline 04D2mh & 09:59:45.872 & $+02: 08: 27.94$ & 2005-01-09 & 2400 & $\mathrm{C}$ & 720 & 1.00 & 22.90 & 730 \\
\hline 04D2mj & $10: 00: 36.535$ & $+02: 34: 37.44$ & 2005-01-09 & 3600 & $N \& S$ & 720 & 0.62 & 23.19 & 54 \\
\hline 05D1az & $02: 25: 12.492$ & $-04: 36: 08.17$ & 2005-09-04 & 5400 & $N \& S$ & 720 & 0.63 & 23.78 & 1246 \\
\hline 05D1by & $02: 24: 35.448$ & $-04: 12: 04.16$ & 2005-09-03 & 5400 & $N \& S$ & 720 & 0.62 & 23.56 & 9 \\
\hline 05D1bz & $02: 25: 00.685$ & $-04: 12: 00.74$ & 2005-09-02 & 7200 & $N \& S$ & 720 & 0.88 & 23.96 & 4310 \\
\hline \multirow[t]{2}{*}{ 05D1cc } & $02: 26: 31.173$ & $-04: 09: 53.40$ & 2005-09-05 & 3600 & $N \& S$ & 720 & 0.46 & 23.18 & 114 \\
\hline & & & 2005-09-06 & 1800 & & & 0.83 & 23.18 & 114 \\
\hline 05D1ee & $02: 24: 46.766$ & $-04: 00: 01.86$ & 2005-10-31 & 3600 & $N \& S$ & 720 & 0.59 & 23.24 & 25 \\
\hline 05D1ej & 02:26:06.317 & $-04: 43: 45.79$ & $2005-10-27$ & 3600 & $N \& S$ & 720 & 0.47 & 22.83 & 15 \\
\hline \multirow[t]{2}{*}{ 05D1em } & 02:24:05.503 & $-04: 56: 23.35$ & 2005-11-05 & 3600 & $N \& S$ & 720 & 0.62 & 23.71 & 476 \\
\hline & & & 2005-11-06 & 1800 & & & 0.57 & 23.71 & 9524 \\
\hline 05D1eo & $02: 25: 23.116$ & $-04: 38: 33.36$ & 2005-10-27 & 5400 & $N \& S$ & 720 & 0.39 & 23.75 & 21 \\
\hline 05D1er & $02: 25: 41.466$ & $-04: 00: 16.32$ & $2005-10-28$ & 7200 & $N \& S$ & 720 & 0.55 & 23.99 & 94 \\
\hline 05D1hn & $02: 24: 36.254$ & $-04: 10: 54.94$ & 2005-12-04 & 2700 & $\mathrm{C}$ & 680 & 0.63 & 20.86 & 249 \\
\hline 05D1ju & $02: 26: 57.303$ & $-04: 08: 02.78$ & $2005-12-27$ & 7200 & $N \& S$ & 720 & 0.50 & 24.04 & 91 \\
\hline 05D1ka & $02: 24: 58.945$ & $-04: 34: 28.56$ & $2005-12-28$ & 5400 & $N \& S$ & 720 & 0.86 & 23.21 & 1059 \\
\hline 05D1kl & $02: 24: 33.544$ & $-04: 19: 08.33$ & 2005-12-31 & 5400 & $N \& S$ & 720 & 0.59 & 23.61 & 247 \\
\hline 05D2ab & 10:01:50.833 & $+02: 06: 23.02$ & 2005-01-09 & 1800 & $\mathrm{C}$ & 680 & 0.67 & 21.83 & 263 \\
\hline 05D2ah & $10: 01: 28.704$ & $+01: 51: 46.18$ & 2005-01-09 & 1800 & $\mathrm{C}$ & 680 & 0.96 & 20.83 & 1017 \\
\hline 05D2ck & 10:00:45.203 & $+02: 34: 22.13$ & $2005-02-16$ & 5400 & $N \& S$ & 720 & 0.65 & 23.88 & 7592 \\
\hline 05D2ja & 10:00:03.809 & $+02: 17: 36.12$ & 2005-05-07 & 5400 & $\mathrm{C}$ & 680 & 0.94 & 22.15 & 115 \\
\hline 05D2nt & 10:00:58.234 & $+02: 22: 21.62$ & $2005-12-26$ & 5400 & $N \& S$ & 720 & 0.69 & 23.46 & 340 \\
\hline 05D2ob & 09:59:00.705 & $+01: 50: 56.62$ & $2005-12-28$ & 5400 & $N \& S$ & 720 & 0.73 & 24.22 & 242 \\
\hline 05D3ax & $14: 19: 17.595$ & $+52: 41: 15.07$ & 2005-02-11 & 5400 & $N \& S$ & 720 & 0.45 & 23.40 & 342 \\
\hline 05D3bt & $14: 18: 35.980$ & $+52: 54: 55.95$ & 2005-03-06 & 3600 & $\mathrm{C}$ & 720 & 0.84 & 23.52 & 71 \\
\hline 05D3cf & $14: 16: 53.369$ & $+52: 20: 42.47$ & 2005-03-08 & 3600 & $\mathrm{C}$ & 720 & 0.69 & 22.96 & 94 \\
\hline 05D3ci & $14: 21: 48.085$ & $+52: 26: 43.33$ & 2005-03-09 & 5400 & $N \& S$ & 720 & 0.76 & 23.03 & 70 \\
\hline $05 \mathrm{D} 3 \mathrm{cq}$ & $14: 18: 46.173$ & $+53: 07: 55.55$ & 2005-04-11 & 5400 & $N \& S$ & 720 & 0.64 & 24.10 & 156 \\
\hline $05 \mathrm{D} 3 \mathrm{cx}$ & $14: 21: 06.560$ & $+52: 45: 01.70$ & 2005-04-09 & 5400 & $N \& S$ & 720 & 0.50 & 23.52 & 65 \\
\hline 05D3jq & $14: 21: 45.462$ & $+53: 01: 47.53$ & 2005-06-04 & 5400 & $N \& S$ & 720 & 0.60 & 23.36 & 30 \\
\hline 05D3ki & $14: 21: 16.341$ & $+52: 35: 42.47$ & 2005-05-14 & 5400 & $N \& S$ & 720 & 0.67 & 23.68 & 49 \\
\hline $05 \mathrm{D} 3 \mathrm{~km}$ & $14: 22: 38.298$ & $+53: 04: 01.14$ & 2005-06-04 & 5400 & $N \& S$ & 720 & 1.0 & 23.89 & 536 \\
\hline 05D3kp & $14: 20: 02.952$ & $+52: 16: 15.28$ & 2005-06-03 & 5400 & $N \& S$ & 720 & 0.81 & 23.36 & 7848 \\
\hline 05D3kt & $14: 19: 53.730$ & $+52: 44: 34.64$ & 2005-06-03 & 5400 & $N \& S$ & 720 & 0.82 & 23.53 & 141 \\
\hline 05D3lb & $14: 17: 31.775$ & $+53: 10: 04.38$ & 2005-06-05 & 5400 & $N \& S$ & 720 & 0.51 & 23.38 & 192 \\
\hline 05D31c & $14: 22: 22.902$ & $+52: 28: 44.11$ & 2005-06-06 & 3600 & $\mathrm{C}$ & 720 & 0.63 & 22.67 & 17 \\
\hline 05D3lw & $14: 17: 44.021$ & $+53: 06: 24.98$ & 2005-07-01 & 7200 & $N \& S$ & 720 & 0.45 & 24.41 & 170 \\
\hline 05D3mn & 14:18:45.206 & $+52: 19: 23.56$ & 2005-07-12 & 7200 & $N \& S$ & 720 & 0.77 & 23.31 & 77 \\
\hline 05D3mh & $14: 18: 59.805$ & $+52: 40: 03.50$ & 2005-07-10 & 7200 & $N \& S$ & 720 & 0.54 & 23.50 & 30 \\
\hline 05D3mq & 14:19:00.398 & $+52: 23: 06.81$ & 2005-08-03 & 1800 & $\mathrm{C}$ & 680 & 0.71 & 21.48 & 4748 \\
\hline 05D3mx & $14: 22: 09.078$ & $+52: 13: 09.35$ & 2005-07-30 & 2700 & $\mathrm{C}$ & 680 & 0.83 & 23.10 & $>10000$ \\
\hline 05D3ne & $14: 21: 02.946$ & $+52: 29: 43.92$ & 2005-07-14 & 2700 & $\mathrm{C}$ & 680 & 0.85 & 20.48 & 1074 \\
\hline 05D4av & $22: 14: 10.515$ & $-17: 54: 42.67$ & 2005-07-10 & 3600 & N\&S & 720 & 0.53 & 23.2 & 77 \\
\hline 05D4bm & 22:17:04.621 & $-17: 40: 39.45$ & 2005-07-11 & 3600 & $\mathrm{C}$ & 680 & 0.55 & 22.04 & 144 \\
\hline 05D4ca & $22: 14: 11.350$ & $-17: 48: 15.47$ & 2005-08-02 & 2700 & $\mathrm{C}$ & 720 & 0.63 & 22.24 & 156 \\
\hline 05D4cn & $22: 13: 31.454$ & $-17: 17: 19.92$ & 2005-08-02 & 5400 & $N \& S$ & 720 & 0.70 & 23.30 & 82 \\
\hline 05D4dt & $22: 14: 25.851$ & $-17: 40: 16.03$ & 2005-09-02 & 3600 & $N \& S$ & 720 & 0.55 & 22.62 & 20 \\
\hline 05D4dx & $22: 13: 39.387$ & $-18: 03: 20.99$ & 2005-09-06 & 5400 & $N \& S$ & 720 & 0.47 & 23.14 & 39 \\
\hline 05D4dy & $22: 15: 30.102$ & $-18: 12: 55.54$ & 2005-09-06 & 5400 & $N \& S$ & 720 & 0.53 & 23.87 & 684 \\
\hline 05D4fn & 22:17:01.076 & $-17: 49: 25.16$ & 2005-10-08 & 7200 & $N \& S$ & 720 & 0.65 & 23.86 & 4474 \\
\hline 05D4fo & $22: 15: 20.925$ & $-17: 16: 05.25$ & 2005-10-07 & 3600 & $N \& S$ & 720 & 0.77 & 22.47 & 1845 \\
\hline 05D4gw & $22: 14: 47.365$ & $-17: 31: 54.99$ & 2005-11-05 & 5400 & $N \& S$ & 720 & 0.46 & 23.69 & 283 \\
\hline 05D4hn & $22: 17: 13.545$ & $-17: 54: 45.40$ & 2005-12-01 & 5400 & $N \& S$ & 720 & 0.50 & 23.50 & 474 \\
\hline 06D3bz & $14: 17: 10.044$ & $+53: 01: 29.31$ & 2006-02-08 & 3600 & $N \& S$ & 720 & 0.71 & 23.20 & 115 \\
\hline 06D3cb & $14: 20: 43.536$ & $+52: 11: 28.11$ & 2006-02-03 & 5400 & $N \& S$ & 720 & 0.88 & 23.61 & 213 \\
\hline 06D3cc & $14: 17: 31.604$ & $+52: 54: 44.79$ & 2006-02-02 & 5400 & $N \& S$ & 720 & 0.58 & 23.55 & 99 \\
\hline 06D3cl & $14: 22: 15.106$ & $+52: 16: 41.53$ & 2006-03-06 & 5400 & $N \& S$ & 720 & 0.61 & 23.75 & 264 \\
\hline 06D3cn & 14:19:25.826 & $+52: 38: 27.78$ & 2006-03-06 & 1800 & $\mathrm{C}$ & 680 & 0.40 & 21.26 & 372 \\
\hline 06D3du & $14: 21: 18.563$ & $+53: 03: 27.10$ & 2006-05-24 & 5400 & $N \& S$ & 720 & 0.45 & 24.27 & 54 \\
\hline 06D3dz & 14:18:14.731 & $+52: 56: 23.11$ & 2006-05-02 & 2400 & $\mathrm{C}$ & 680 & 0.98 & 22.19 & 66 \\
\hline
\end{tabular}




\section{Appendix A: Results - Spectroscopic analysis of SNLS SNe la}

Table A.1. Equivalent width and ejection velocity results for the published high redshift SNe Ia observed by the SNLS at the Gemini telescopes. This set includes all of the SNLS objects (reduced by the author) that were confirmed as Type Ia SNe (a confidence index of 3 or higher) and were subject to less than $65 \%$ contamination from their host galaxies. The blank spaces ("...") indicate where a measurement had to be removed due to inadequate wavelength coverage. The uncertainties in the $E W$ results include measurement errors, errors from any possible variance in the pseudo-continuum, and the uncertainty from the host galaxy contamination correction.

\begin{tabular}{|c|c|c|c|c|c|}
\hline Name & Day $^{a *}$ & $E W\{\mathrm{CaII}\}^{b *}$ & $E W\{\mathrm{SiII}\}^{b *}$ & $E W\{\mathrm{MgII}\}^{b *}$ & $v_{\mathrm{ej}}^{c *} / 1000.0$ \\
\hline 03D1ax & $-2.30(0.11)$ & $117.6(15.4)$ & $13.8(2.0)$ & $105.3(10.0)$ & $11.84(0.29)$ \\
\hline 03D1bk & $-5.23(0.25)$ & 95.7(11.6) & $21.0(3.7)$ & $98.3(18.2)$ & $12.71(3.40)$ \\
\hline 03D1cm & $-4.42(0.59)$ & $52.0(13.1)$ & $14.8(10.6)$ & $125.0(27.5)$ & $16.98(1.81)$ \\
\hline 03D1co & $7.42(0.44)$ & $87.8(16.9)$ & $16.7(3.4)$ & $246.2(21.8)$ & $13.99(6.45)$ \\
\hline 03D1ew & $1.53(0.91)$ & $144.2(19.5)$ & $23.5(12.7)$ & $83.2(26.4)$ & $20.21(1.32)$ \\
\hline 03D4cj & $-8.05(0.01)$ & & $5.9(6.1)$ & $131.6(13.8)$ & \\
\hline 03D4cn & $0.53(2.42)$ & $116.9(16.0)$ & $17.0(5.4)$ & $76.0(12.3)$ & $13.85(1.14)$ \\
\hline 03D4cy & $5.86(0.59)$ & $163.2(22.5)$ & $8.7(5.8)$ & $124.5(35.3)$ & $16.38(3.22)$ \\
\hline 03D4fd & $-0.88(0.70)$ & $100.2(14.3)$ & $21.4(5.2)$ & 79.4(16.4) & $14.83(0.45)$ \\
\hline 03D4gl & $8.27(0.28)$ & $104.8(16.6)$ & $17.0(5.3)$ & $72.9(10.4)$ & $20.42(1.96)$ \\
\hline 04D1de & $-6.80(0.10)$ & $91.4(14.5)$ & $9.4(1.3)$ & $105.1(12.9)$ & $20.78(1.01)$ \\
\hline 04D1hd & $1.85(0.04)$ & $\ldots$ & $11.9(11.5)$ & 99.1(21.4) & $15.25(1.44)$ \\
\hline 04D1hy & $-2.19(0.44)$ & $\cdots$ & $15.5(6.0)$ & $101.4(16.9)$ & $16.11(2.05)$ \\
\hline 04D1ow & $6.28(0.42)$ & $139.0(14.9)$ & $21.1(10.2)$ & $92.7(22.4)$ & $14.66(3.02)$ \\
\hline 04D2ae & $-1.95(1.78)$ & $\ldots$ & 13.1(7.5) & $128.4(18.7)$ & $19.41(1.87)$ \\
\hline 04D2mh & $1.58(0.24)$ & $116.5(29.1)$ & $18.2(11.9)$ & 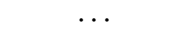 & $14.41(5.15)$ \\
\hline 04D3dd & 3.68 (1.69) & $109.5(19.8)$ & $30.7(15.9)$ & $\cdots$ & $16.97(1.42)$ \\
\hline 04D3fq & $1.35(0.66)$ & 83.3(23.4) & $26.6(10.2)$ & $102.5(26.2)$ & $16.26(2.76)$ \\
\hline 04D3kr & $5.37(0.09)$ & $\ldots$ & $15.8(1.8)$ & $118.0(11.6)$ & $14.43(1.63)$ \\
\hline 04D3lp & $1.00(0.20)$ & $100.1(16.9)$ & $\ldots$ & $\ldots$ & $17.62(2.28)$ \\
\hline 04D3mk & $-1.47(0.55)$ & $\ldots$ & $2.2(1.0)$ & $129.0(15.4)$ & $12.31(2.08)$ \\
\hline 04D3ml & $-0.89(0.84)$ & $96.8(11.6)$ & $\ldots$ & $138.9(25.3)$ & $13.06(1.53)$ \\
\hline 04D3nq & $9.36(0.04)$ & $\ldots$ & $\cdots$ & $154.5(34.8)$ & $\ldots$ \\
\hline 04D3ny & $2.15(0.67)$ & $\cdots$ & $26.9(7.4)$ & 91.3(18.8) & $11.23(4.32)$ \\
\hline 04D4dm & $3.55(0.47)$ & $\ldots$ & 9.7(4.9) & $120.6(24.5)$ & $18.56(2.50)$ \\
\hline 04D4hu & $6.22(0.29)$ & $68.6(11.2)$ & $12.9(3.0)$ & $\ldots$ & $\ldots$ \\
\hline 04D4ic & $2.92(0.66)$ & $\ldots$ & $17.7(7.9)$ & $137.0(21.3)$ & $15.92(1.69)$ \\
\hline 04D4ii & $-4.58(0.34)$ & $\cdots$ & $10.7(3.9)$ & $122.2(15.3)$ & $19.99(3.21)$ \\
\hline 04D4jy & $2.00(0.40)$ & $73.9(24.8)$ & $\ldots$ & $\ldots$ & $15.34(1.48)$ \\
\hline 05D1az & $8.85(0.17)$ & $75.6(13.5)$ & $8.9(7.0)$ & $102.1(17.5)$ & $14.13(0.15)$ \\
\hline 05D1cc & $6.80(0.15)$ & 89.7(17.9) & $\ldots$ & 102.0(11.8) & $12.79(0.58)$ \\
\hline 05D1em & $6.69(0.23)$ & $126.8(17.7)$ & $25.0(16.5)$ & $140.9(34.2)$ & $14.95(2.76)$ \\
\hline 05D1er & $2.83(0.42)$ & $\ldots$ & $29.0(5.5)$ & $160.7(18.9)$ & $16.35(1.83)$ \\
\hline 05D1ju & $6.28(0.27)$ & $163.2(24.6)$ & $13.2(3.6)$ & $116.50(15.7)$ & $18.46(2.79)$ \\
\hline 05D1kl & $-4.27(0.21)$ & $97.0(17.0)$ & $7.6(5.3)$ & $100.8(14.9)$ & $18.92(3.56)$ \\
\hline 05D2ab & $-1.30(0.08)$ & $\ldots$ & $13.8(7.5)$ & $93.5(11.3)$ & $15.34(3.64)$ \\
\hline 05D2ah & $-2.49(0.03)$ & $\cdots$ & $10.2(10.4)$ & $92.0(21.0)$ & \\
\hline 05D2ck & $0.730(0.74)$ & $71.5(24.7)$ & $24.4(8.3)$ & $148.7(24.0)$ & $14.07(0.36)$ \\
\hline 05D2ja & $9.63(0.12)$ & $\ldots$ & $\ldots$ & $161.3(25.3)$ & $\ldots$ \\
\hline 05D3ax & $8.03(0.39)$ & $118.9(9.0)$ & $\cdots$ & $65.6(7.2)$ & $14.77(0.62)$ \\
\hline 05D3cf & $13.68(0.41)$ & $\ldots$ & $\cdots$ & 273.1(44.1) & $\ldots$ \\
\hline $05 \mathrm{D} 3 \mathrm{cq}$ & $11.6(1.35)$ & $106.9(23.1)$ & $\ldots$ & $\ldots$ & $13.51(0.32)$ \\
\hline $05 \mathrm{D} 3 \mathrm{cx}$ & $10.28(0.61)$ & $\ldots$ & $27.2(5.7)$ & $98.5(13.6)$ & $10.01(0.33)$ \\
\hline $05 \mathrm{D} 3 \mathrm{~km}$ & $1.46(0.31)$ & 87.1(19.7) & $\ldots$ & $\ldots$ & $13.67(2.07)$ \\
\hline 05D3kt & $-3.86(0.30)$ & $\ldots$ & $\cdots$ & $123.5(15.2)$ & $18.34(2.68)$ \\
\hline $05 \mathrm{D} 3 \mathrm{lb}$ & $-1.21(0.24)$ & $\ldots$ & $9.2(2.6)$ & $88.5(7.7)$ & $19.16(1.35)$ \\
\hline $05 \mathrm{D} 3 \mathrm{mh}$ & $2.71(0.40)$ & $96.4(22.1)$ & $25.6(4.6)$ & $154.3(21.5)$ & $13.43(1.79)$ \\
\hline 05D3mn & $2.82(0.40)$ & $80.7(25.7)$ & $14.8(3.7)$ & $136.4(14.7)$ & $13.88(0.24)$ \\
\hline $05 \mathrm{D} 3 \mathrm{mq}$ & $8.45(0.07)$ & $\ldots$ & $3.6(15.1)$ & $232.8(35.2)$ & $\ldots$ \\
\hline 05D4av & $5.84(0.15)$ & $\cdots$ & $9.3(6.7)$ & $54.1(9.5)$ & $\cdots$ \\
\hline 05D4bm & $-3.81(0.05)$ & $162.0(14.3)$ & $14.5(2.9)$ & 85.7(7.8) & $19.98(0.99)$ \\
\hline 05D4cn & $0.39(0.32)$ & $62.3(10.7)$ & $18.2(4.3)$ & $146.2(13.2)$ & $14.10(0.57)$ \\
\hline 05D4dy & $2.13(0.55)$ & . & $23.2(6.6)$ & $127.1(19.2)$ & $11.33(2.50)$ \\
\hline 05D4gw & $0.13(0.34)$ & $162.6(22.1)$ & $16.8(5.9)$ & $115.8(13.8)$ & $18.70(2.08)$ \\
\hline 05D4fo & $-5.31(0.03)$ & $\ldots$ & $28.6(5.1)$ & $82.6(11.7)$ & $\ldots$ \\
\hline
\end{tabular}

\footnotetext{
${ }^{a}$ Rest-frame day, relative to $B^{\prime} \max$;

${ }^{b}$ Equivalent Width, $\AA$;

${ }^{c}$ Ejection velocity, $\mathrm{km} \mathrm{s}^{-1}$;

* $1 \sigma$ errors listed in parentheses.
} 


\section{Appendix B: SNLS observed spectra}

Sprectra from each Supernova Legacy Survey SN candidate observed at the Gemini telescopes from December 2004 through May 2006. The observed properties and observation setup for each object is summarized in Table 6 and the final object identifications and redshift estimates are displayed in Table 7. These spectra have been corrected for sky absorption, the narrow host galaxy features have been removed, and the GMOS chip gaps have been interpolated over. These images show the observed spectrum, re-binned to $7.5 \AA$ resolution, in dark blue with a smoothed version of the spectrum overplot in light blue. The smoothed spectrum is for illustrative purposes only and was generated witha Savitsky-Golay filter computed with a 3rd degree polynomial fit over 40 pixels of the observed spectrum. Note that for some of these spectra a large amount of host galaxy contamination is clear. 
T. J. Bronder et al.: SNLS spectroscopy: testing for evolution in type Ia supernovae, Online Material p 5
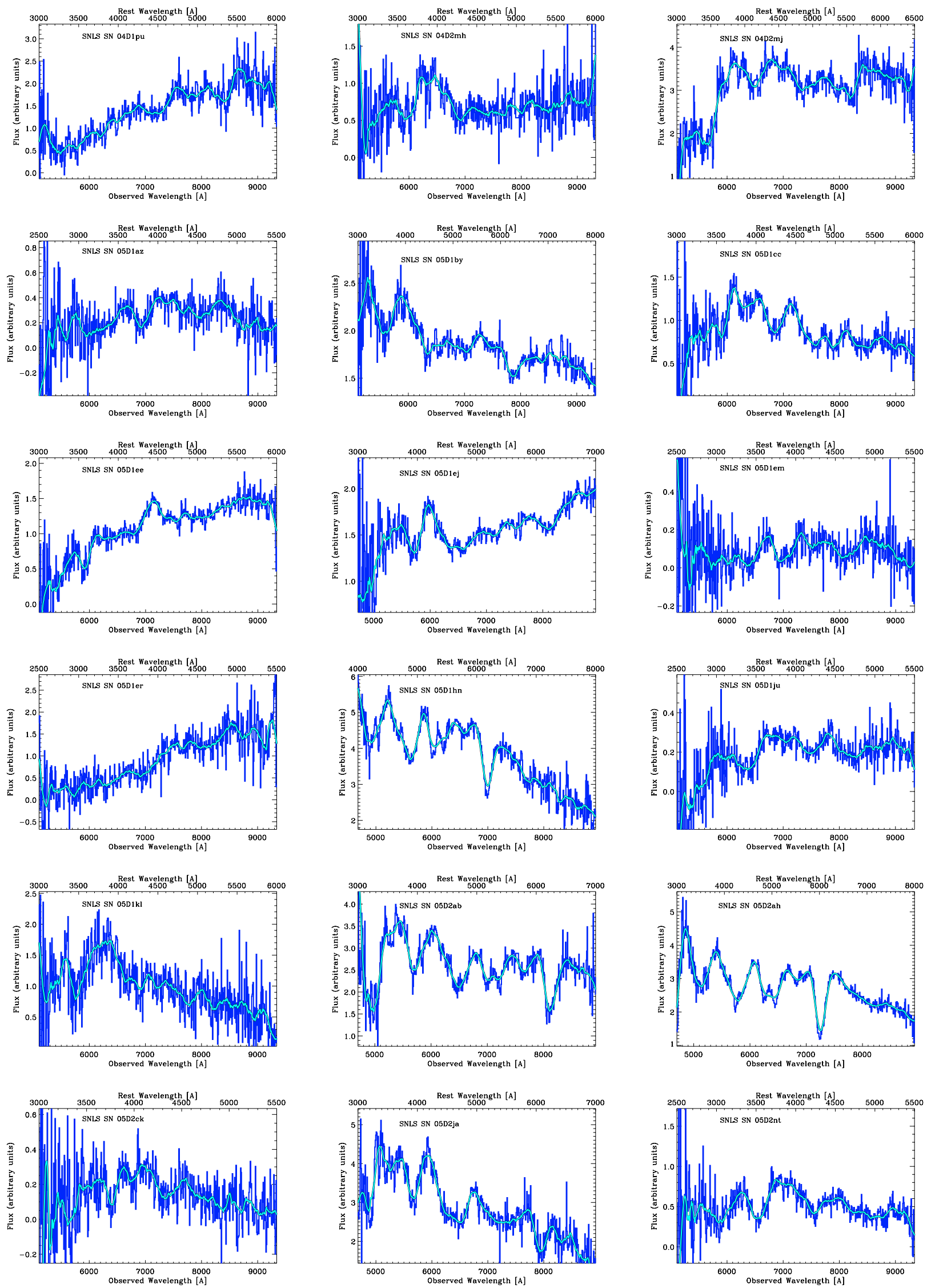

Fig. B.1. Confirmed SNe Ia. The observed, re-binned spectrum is shown in dark blue and the smoothed spectrum is overplot in light blue for illustrative purposes. 
T. J. Bronder et al.: SNLS spectroscopy: testing for evolution in type Ia supernovae, Online Material p 6
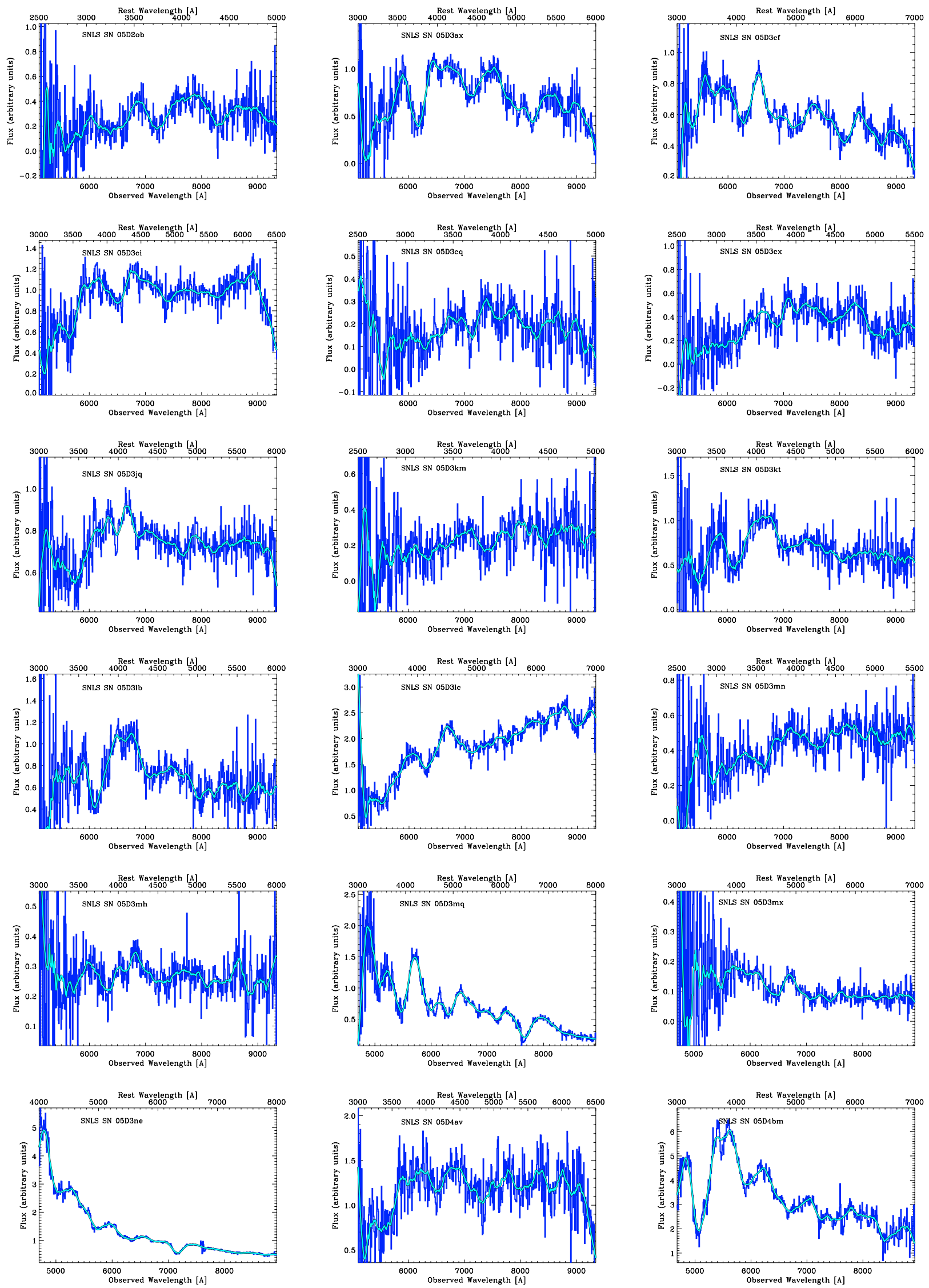

Fig. B.2. Confirmed SNe Ia. The observed, re-binned spectrum is shown in dark blue and the smoothed spectrum is overplot in light blue for illustrative purposes. 
T. J. Bronder et al.: SNLS spectroscopy: testing for evolution in type Ia supernovae, Online Material $p 7$
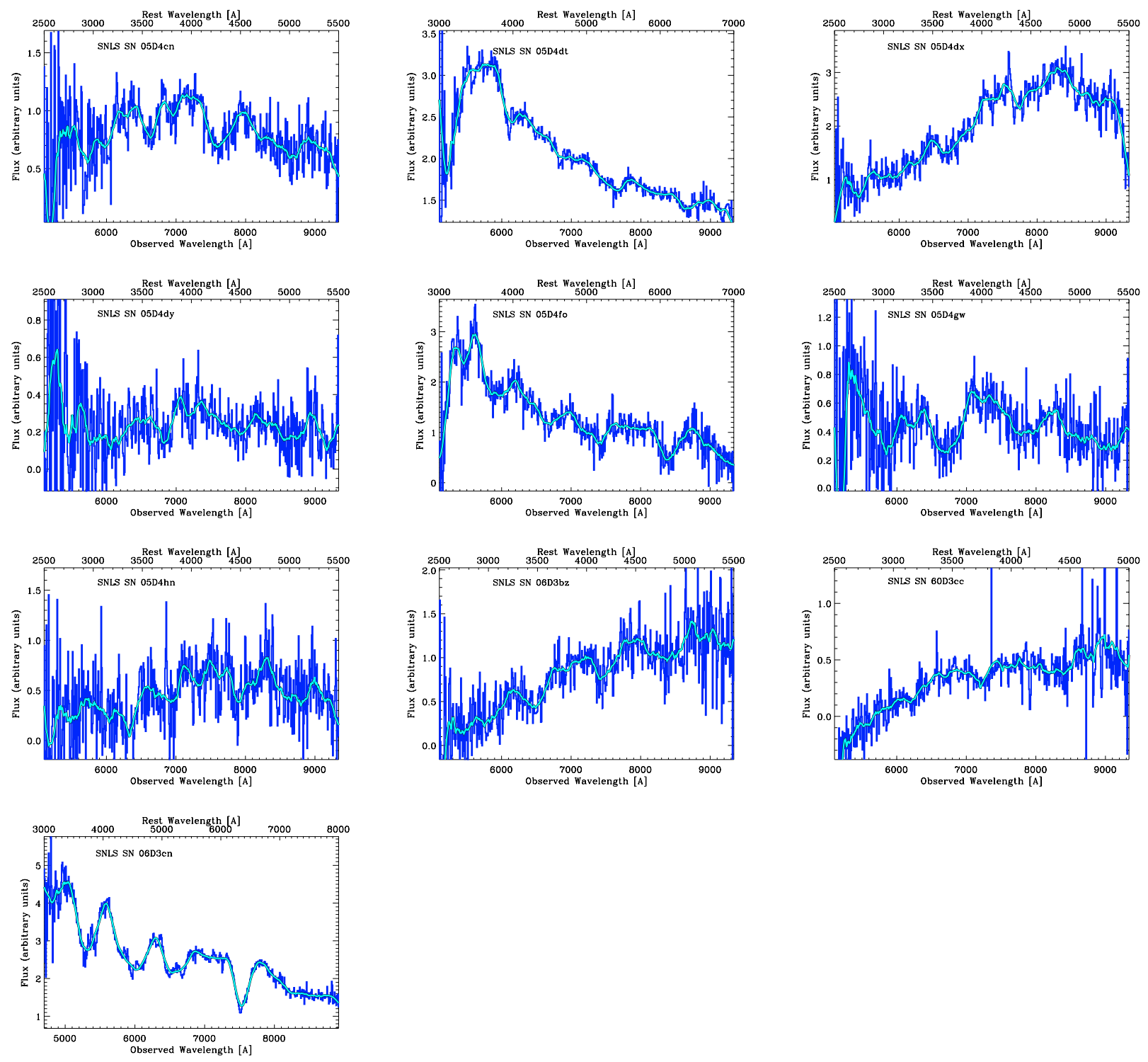

Fig. B.3. Confirmed SNe Ia. The observed, re-binned spectrum is shown in dark blue and the smoothed spectrum is overplot in light blue for illustrative purposes. 
T. J. Bronder et al.: SNLS spectroscopy: testing for evolution in type Ia supernovae, Online Material $p 8$
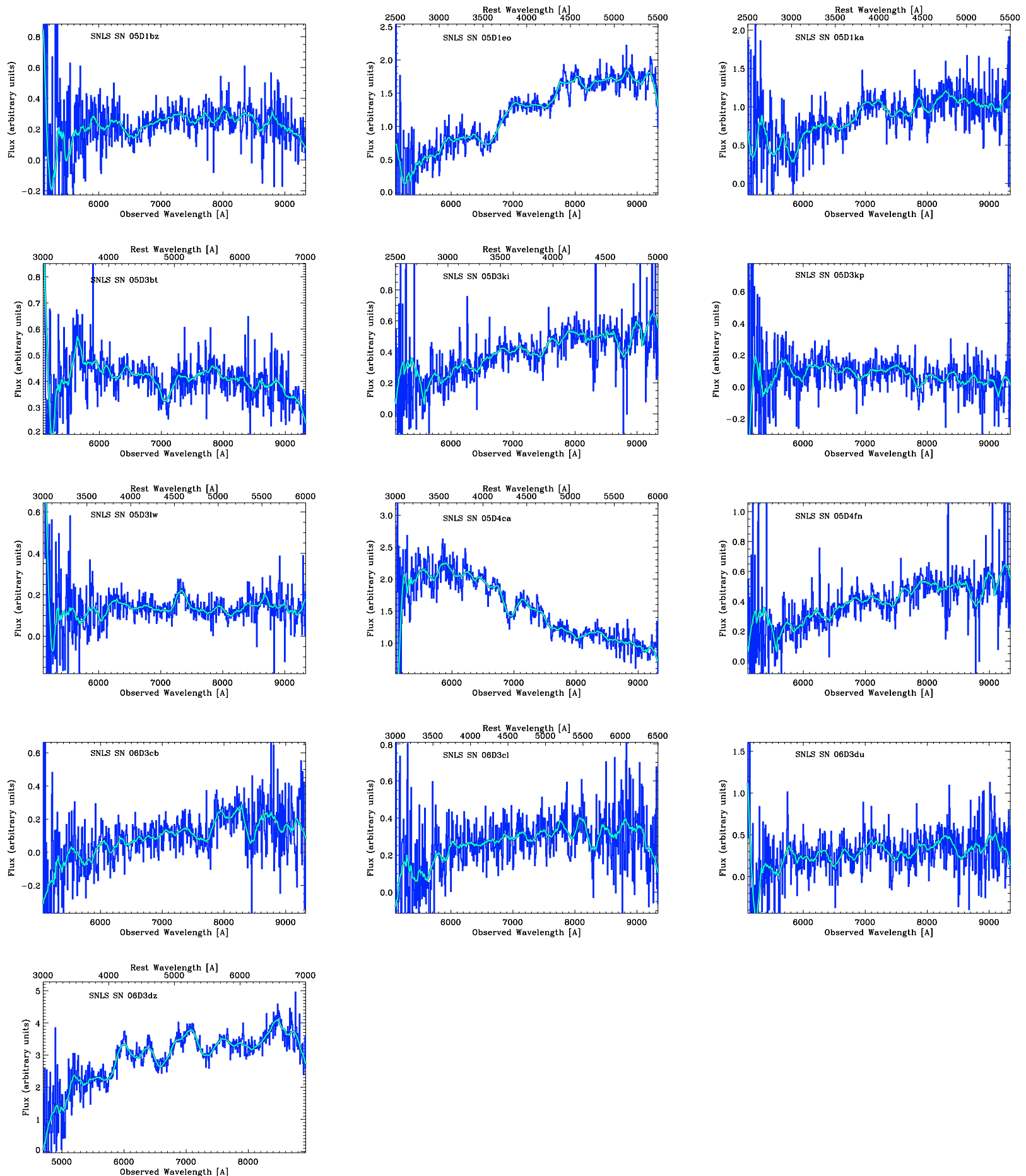

Fig. B.4. Unidentified SN candidates. The observed, re-binned spectrum is shown in dark blue and the smoothed spectrum is overplot in light blue for illustrative purposes. 